\title{
Prediction of viscous cracking and cyclic fatigue of salt polycrystals using a joint-enriched Finite Element Model
}

\author{
Cheng Zhu ${ }^{\mathrm{a}}$, Ahmad Pouya ${ }^{\mathrm{b}}$, Chloé Arson ${ }^{\mathrm{a}}$ \\ ${ }^{a}$ School of Civil and Environmental Engineering, Georgia Institute of Technology, \\ Atlanta, USA \\ ${ }^{b}$ Laboratoire Navier (IFSTTAR, CNRS, ENPC), Paris-Est University, Champs sur \\ Marne, France
}

\begin{abstract}
We present a new Joint-enriched Finite Element Method (JFEM) to predict viscous damage and fatigue in halite polycrystals in 2D. Different viscoplastic finite elements are used to represent grains of different orientations, and joint elements are used for modeling crack propagation. Simulations of uniaxial creep tests show that, as it could be predicted theoretically, viscous shear deformation in grains causes geometric incompatibilities. Numerical results also show that the transition between secondary and tertiary creep corresponds to inter-granular crack coalescence. The JFEM model captures the mechanical behavior of halite under cyclic loading, mainly: (a) Higher stress amplitude, lower confining stress, and lower loading frequency increase deformation and damage; (b) The polycrystal's Young's modulus decreases exponentially with the number of cycles; (c) The behavior is similar for different loading directions. Simulations with intra- and inter- granular joint elements show that most stress concentrations occur in intra-granular joints where several angular grains are in contact. Results of creep tests obtained with the JFEM are compared to those obtained with an inclusion-matrix model that accounts for damage accommodation due to grain breakage. Both the JFEM and inclusion-matrix models are calibrated against experimental creep tests to: (a) Produce a Young's modulus of 23 GPa for the polycrystal; (b) Match secondary creep strain rates; (c) Match the time of tertiary creep initiation. In the inclusion-matrix model, the absence of grain geometric rearrangement results in a brutal failure just after the first grain breakage that triggers tertiary creep. Moreover, the JFEM model highlights the development of crack patterns upon viscous deformation. The JFEM is of
\end{abstract}


great promise to understand complex phenomena of viscous accommodation coupled with grain interface debonding.

Keywords: salt polycrystal, self-consistent method, Finite Element Method, creep test, cyclic loading, damage

\section{Introduction}

Energy demand, environment protection, and public health preservation raise pressing needs for safe and sustainable geological storage systems for energy (e.g., compressed air, petroleum, and hydrogen) and waste (e.g., nuclear waste, carbon dioxide). Salt rock has been used as a host medium because of its favorable creep properties, low gas permeability, self-healing capabilities, and solubility in water. Halite $(\mathrm{NaCl})$ is a viscous material that is subject to fatigue under cyclic loading [1]. Constitutive models were proposed for salt rock (e.g., [2, 3, 4]), which allowed the long-term performance assessment of salt caverns (e.g., [5, 6, 7, 8]). Fundamental deformation processes and flow properties of salt were studied experimentally at the crystal scale [9]. But few studies relate the local behavior of grains to the evolution of the structure of the polycrystal and to salt macroscopic behavior.

Microstructure-enriched models were used to understand the influence of grain size, orientation, shape, and boundary topology in polycrystalline materials (e.g., [10]). Finite Element models assumed simplified grain shapes such as a squared or a cubic mesh, in which each element corresponds to a specific grain orientation $[11,12,13]$. Such methods are not based on a realistic representation of microstructure and cannot explain the fundamental processes that drive the macroscopic behavior of the polycrystal.

Finite Element Methods (FEM) with discontinuities were proposed to predict the deformation and strength of polycrystals at various scales in 2D, extended 2D, or 3D (e.g., [14]). Crack propagation within polycrystals was simulated with Cohesive Zone Models (CZM) [15, 16, 17, 18] and extended Finite Element Methods (XFEM) [19] enriched with jump interpolation functions. Microstructure-based FEM simulations were used to study metallic materials such as aluminum [20], copper [21, 14], interstitial-free (IF) steel [22], and a variety of alloys [23, 24]. To date however, FEM models cannot explain the time-dependent viscous damage and fatigue behavior of polycrystals subject to long-term creep or cyclic loading. Only a few studies are 
based on real grain structures and account for various damage mechanisms including inter- and intra- granular crack propagation [24].

In this paper, we present a new Joint-enriched Finite Element Method (JFEM) to predict viscous damage and fatigue in halite polycrystals in 2D. Different visco-plastic finite elements are used to represent grains of different orientations, and a Cohesive Zone Model (CZM) is used for the joint elements that represent interfaces. Inter-granular damage at the grain-matrix interface is a measure of the progressive accumulation of microcracking and explains the influence of geometrical incompatibilities on the macroscopic material response. The CZM can predict both mode I and mode II crack propagation. We used POROFIS FEM software [25] to simulate uniaxial creep tests and cyclic loading tests. In Section 2, we start by describing the deformation mechanisms of a single crystal of salt. Section 3 explains the Joint-enriched FEM model designed in POROFIS. Section 4 presents the JFEM results obtained with inter-granular interfaces only, and with both inter- and intra- granular interfaces. In Section 5, we compare the JFEM to a homogenization scheme based on the self-consistent method, in which we adapt an inclusion-matrix model of grain breakage that we developed previously in 3D [1] to two-dimensional stress analysis. Both the JFEM and inclusion-matrix models are calibrated against the same experimental creep tests. We explain the differences between the two models, and we discuss the capabilities of state-of-the-art homogenization schemes in describing texture evolution and predicting strain cracking damage.

\section{Single-crystal deformation mechanisms}

In polycrystalline halite, single crystals have different crystallographic orientations and a reduced number of preferential slip systems, which originates geometric incompatibilities. Resulting plastic strains play a dominant role in the determination of internal stresses [26]. In this context, the elastic anisotropy of the $\mathrm{NaCl}$ single crystal has no significant effect. For this reason, almost all the studies on polycrystalline halite are based on the assumption that grains are isotropic in the elastic regime. Anisotropy is only accounted for in the expression of plastic or viscous deformation (e.g., [27, 28]). Thus in the following, we considered an isotropic elastic behavior for single crystalline grains, and, for simplicity, we represented damage by a scalar variable.

We assume that the total strain rate is the sum of the elastic strain rate 
and the viscoplastic strain rate:

$$
\dot{\varepsilon}=\dot{\varepsilon}^{e}+\dot{\varepsilon}^{v p}
$$

In most polycrystalline materials including salt, slip is the primary mechanism for fatigue [29]. The viscoplastic strain rate is calculated from the shear strain rates induced by all possible slip systems:

$$
\begin{gathered}
\dot{\varepsilon}_{i j}^{v p}=\sum \dot{\gamma}^{l} a_{i j}^{l} \\
a_{i j}^{l}=\frac{1}{2}\left(n_{i}^{l} m_{j}^{l}+m_{i}^{l} n_{j}^{l}\right)
\end{gathered}
$$

in which $\dot{\gamma}^{l}$ is the rate of viscoplastic strain of grains subjected to the $l^{\text {th }}$ slip mechanism, and $\boldsymbol{m}^{\boldsymbol{l}}$ and $\boldsymbol{n}^{\boldsymbol{l}}$ are the orthonormal unit vectors that define the slip direction and the direction normal to the slip plane of the $l^{\text {th }}$ slip system, respectively. In 3D, halite crystals present a face-centered cubic (FCC) structure. Slip mechanisms during grain shear deformation occur along six preferential planes, which provides two independent slip systems [1]. Based on the correlations established in [30, 31], we assume that the irreversible shear deformation obeys a power law:

$$
\dot{\gamma}^{l}=\gamma_{0} h^{l}\left|\frac{\tau^{l}}{\tau_{0}}\right|^{n}
$$

in which $n$ and $\gamma_{0}$ are material parameters, representing the sensitivity of the slip rate and the reference strain rate. $\tau_{0}$ is a reference shear stress, arbitrarily set equal to $1 \mathrm{MPa}$. $h^{l}$ depends on the sign of $\tau^{l}: h^{l}=1$ if $\tau^{l}$ is positive; $h^{l}=-1$ if $\tau^{l}$ is negative. In the following, we calibrate the $\gamma_{0}$ and $n$ parameters of the viscoplastic model.

In plane strain, all representative salt crystal orientations are contained in the plane, i.e. representative grain orientations are obtained by rotation about the axis normal to the plane under study, as shown in Fig. 1. 


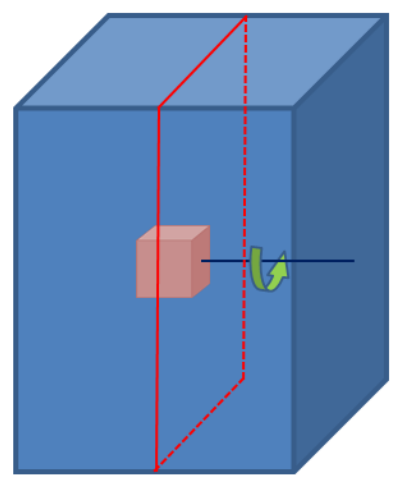

Figure 1: 2D salt polycrystal: representative halite crystal orientations are obtained by varying only one degree of freedom, the angle of rotation $\theta$ about the axis normal to the plane.

In a two-dimensional plane strain case, two of the six salt slip systems control shear deformation (Fig. 2). The two slip systems are oriented by an angle of 90 degrees. We note $\boldsymbol{N}$ and $\boldsymbol{M}$ the normal vector and the gliding vector in the local coordinate system of the grain, respectively.

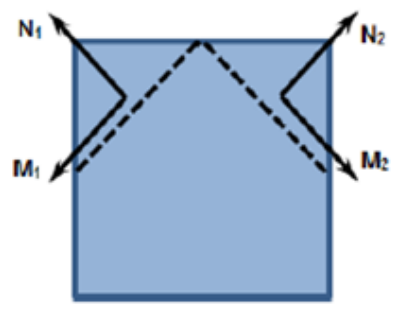

Figure 2: Representation of the two slips systems that control shear deformation at the grain scale in $2 \mathrm{D}$ plane strain $(\boldsymbol{N}$ and $\boldsymbol{M}$ are the normal vector and gliding vector, respectively).

Given a specific angle $\theta$, the two possible slip systems in the global coordinate system $\left(\boldsymbol{n}^{\mathbf{1}}, \boldsymbol{m}^{\mathbf{1}}\right)$ and $\left(\boldsymbol{n}^{\mathbf{2}}, \boldsymbol{m}^{\mathbf{2}}\right)$ are:

$$
\begin{aligned}
& \boldsymbol{n}^{\mathbf{1}}=\left(\begin{array}{c}
\cos \left(\theta+\frac{\pi}{4}\right) \\
\sin \left(\theta+\frac{\pi}{4}\right)
\end{array}\right), \quad \boldsymbol{m}^{\mathbf{1}}=\left(\begin{array}{c}
\cos \left(\theta+\frac{3 \pi}{4}\right) \\
\sin \left(\theta+\frac{3 \pi}{4}\right)
\end{array}\right) \\
& \boldsymbol{n}^{\mathbf{2}}=\left(\begin{array}{c}
\cos \left(\theta+\frac{3 \pi}{4}\right) \\
\sin \left(\theta+\frac{3 \pi}{4}\right)
\end{array}\right), \quad \boldsymbol{m}^{\mathbf{2}}=\left(\begin{array}{c}
\cos \left(\theta+\frac{\pi}{4}\right) \\
\sin \left(\theta+\frac{\pi}{4}\right)
\end{array}\right)
\end{aligned}
$$




\section{Polycrystal Finite Element model}

\subsection{Joint element model}

We use POROFIS [25] to design a numerical model in which salt grains are modeled with Finite Elements (FE), and cracks are modeled with joint elements. Grain FEs are assigned the single-crystal visco-plastic behavior described in Section 2, and different crystal orientations are accounted for by using FEs with different slip mechanisms (see Section 3.2). Joint elements are based on a Cohesive Zone Model (CZM), in which strength evolves with damage $(D)$ according to the model presented in [32], as follows:

$$
F(\sigma, D)=\tau^{2}-\sigma_{n}^{2} \tan ^{2} \eta+2 g(D) \sigma_{c} \sigma_{n}-g^{2}(D) C^{2}
$$

in which $C$ and $\eta$ are respectively the cohesion and the friction angle of intact rock joints. Stresses at joint faces $(\boldsymbol{\sigma})$ are related to the joint stiffness $(\boldsymbol{k})$ and to the joint relative displacement $(\boldsymbol{u})$ by the following elastic damage law:

$$
\boldsymbol{\sigma}=(1-D) \boldsymbol{k} \cdot \boldsymbol{u}
$$

The expression of $g(D)$ is [32]:

$$
g(D)=(1-D)(1-\beta \ln (1-D))
$$

in which $g(D=0)=1$ represents the initial intact state and $g(D=1)=0$ represents the completely damaged state (i.e. ultimate failure). $\sigma_{c}$ is a constant related to $C, \eta$, and to the tensile strength $\sigma_{R}$ of the intact rock joint by the following relation:

$$
\sigma_{c}=\frac{C^{2}+\sigma_{R}^{2} \tan ^{2} \eta}{2 \sigma_{R}}
$$

in which $\sigma_{c}$ is an auxiliary stress value related to the shape of the yield surface (Fig. 3). When $g=1$, we obtain the outer hyperbolic surface. The asymptotic behavior under high compressive stresses corresponds to a conical surface that forms an angle $\eta$ with the $\sigma_{n}$ axis. When damage increases, the yield surface moves towards the interior while the friction angle $\eta$ remains the same. For the limiting case of a completely damaged joint with $g=0$, the frictional fracture has zero cohesion and the friction angle $\eta$ satisfies the following criterion:

$$
F\left(\tau, \sigma_{n}\right)=\tau^{2}-\sigma_{n}^{2} \tan ^{2} \eta
$$


As can be seen in Fig. 3, the tensile strength $\sigma_{R}$ and the cohesion parameter $C$ have to satisfy the following inequality:

$$
\sigma_{R} \tan (\eta)<C
$$

Under a normal tensile stress, the cohesive joint remains undamaged until a critical displacement value $u_{0}$ is reached. The corresponding damage $D$ is assumed to depend exponentially on the relative displacement:

$$
D=1-e^{-\left(u-u_{0}\right) / \beta u_{0}}
$$

in which the constant parameter $\beta$ characterizes the material ductility (the smaller $\beta$, the more brittle the material behavior). Note that damage models similar to that presented in Eq. 13 are often used for interfaces in cementitious materials [33].

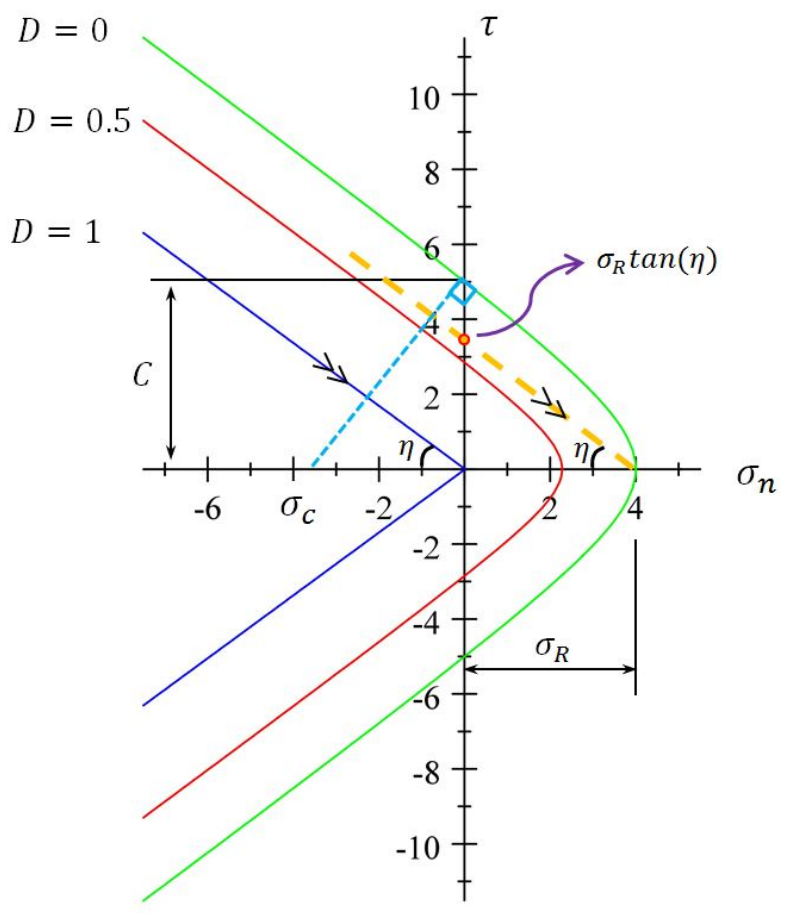

Figure 3: Evolution of the damage-plasticity criterion used in joint elements, from the intact state to the completely damaged state. The yellow dashed line is parallel to the line that represents the limiting criterion when $D=1$, and intersects the $\tau$ axis at $\sigma_{R} \tan (\eta)$. 


\subsection{Model configuration}

In a previous work [34], POROFIS was used by the authors to model a single inclusion embedded in a homogeneous medium. In this study, we aim to model a polycrystal made of a representative number of inclusions (i.e., grains). However, 3D images of halite microstructure are scarce in the literature. Several methods can be used to represent crystal orientations in 2D micrographs, including optical and electron microscopy, automated Electron BackScatter Diffraction (EBSD) and serial sectioning techniques [35]. In this paper, we construct a 2D FEM polycrystal model based on the EBSD map of the salt specimen studied in [36]. Crystal orientations are represented by different colors in the EBSD map (Fig. 4).

We use the software Plot Digitizer to extract grain boundaries from the EBSD map, and we export them in GiD FEM pre- and post-processor [37] in order to construct the mesh. For the sake of simplicity, we select only 12 grain orientations for this 2D model (in which only angle $\theta$ varies, see Fig. 1). These 12 grain FE orientations are uniformly distributed in the interval $\left[\cos (\theta=0)=0, \cos \left(\theta=\frac{\pi}{2}\right)=1\right]$, so as to approximately match the orientations represented in the EBSD map. Our mesh contains a relatively isotropic and uniform distribution of grains with various orientations, which is a realistic representation of a salt polycrystal. We construct two FEM models with: (1) Joint elements that are only located along the grain boundaries (inter-granular cracks); and (2) Joint elements that are located both between and inside the grains (inter- and intra- granular cracks). In both models, the mesh comprises 3,368 surface elements, which are all 0.2 $\mathrm{mm}$ in size (Fig. 5). In model (2), inter-granular and intra-granular joint elements follow the same behavior (Section 3.1), but have different constitutive parameters (Fig. 6). 

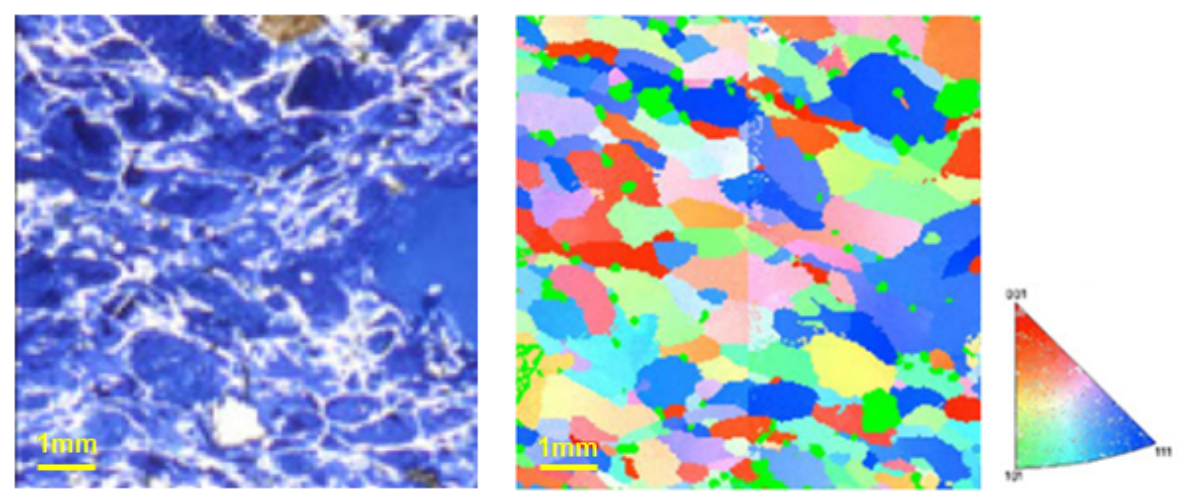

Figure 4: Salt thin sections (adapted from [36]): Microstructure (left) and Automated EBSD map (right).

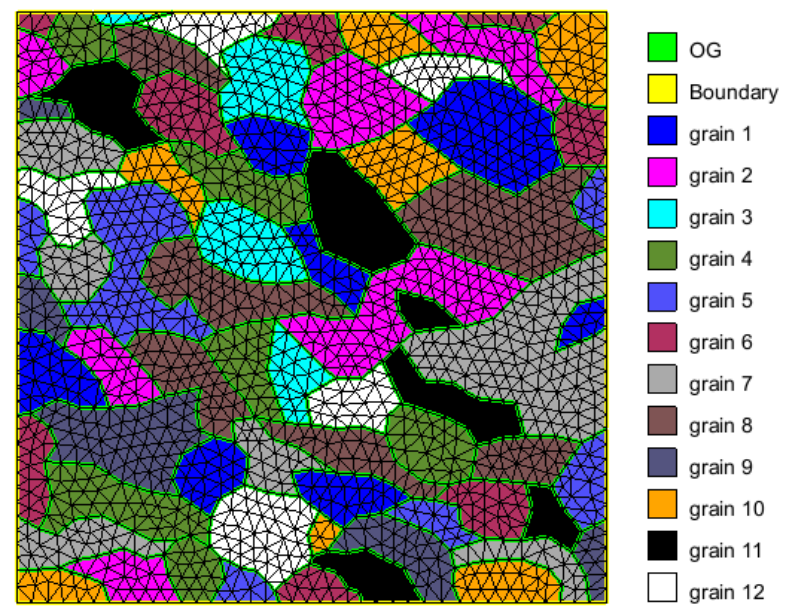

Figure 5: POROFIS FEM model with inter-granular joints only $(8 \mathrm{~mm} \times 8 \mathrm{~mm})$. In the legend, grain numbers refer to grain orientations (12 orientations considered in total), and OG is the color used for joint elements. 


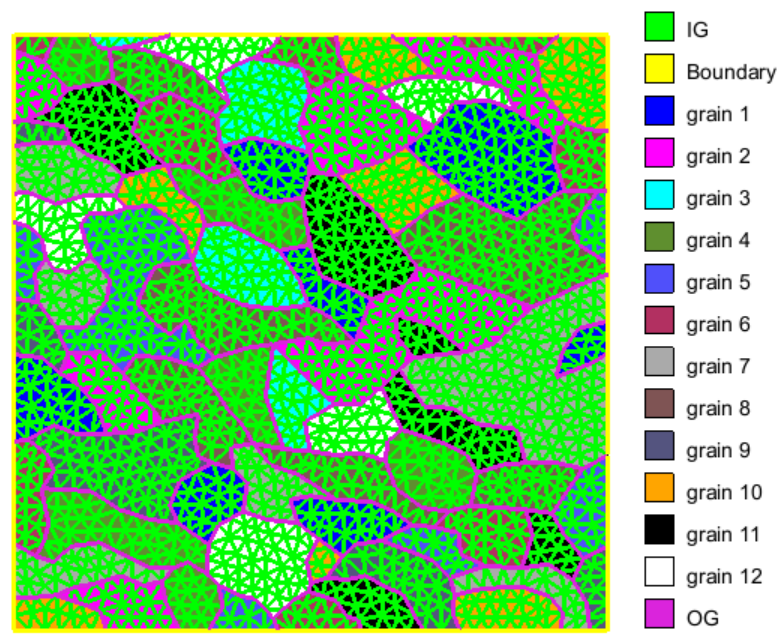

Figure 6: POROFIS FEM model with inter-granular and intra-granular joints $(8 \mathrm{~mm} \times$ $8 \mathrm{~mm}$ ). In the legend, grain numbers refer to grain orientations (12 orientations considered in total), OG is the color used for inter-granular joint elements, and IG is the color used for intra-granular joint elements.

\subsection{Joint-enriched FEM model calibration}

The POROFIS model requires the calibration of: (a) the elastic properties and viscous parameters of the grain Finite Elements; (b) the stiffness and damage parameters of the joint elements. For the elastic properties of singlecrystals (grain FEs), we take a Young's modulus of $43 \mathrm{GPa}$ (in reference to experimental data published in [38]) and a Poisson's ratio of 0.3 (in reference to experimental data published in [39]). Note that the stiffness of a singlecrystal is greater than that of halite polycrystal (typically, $23 \mathrm{GPa}$ according to [39]). This statement is true in other materials such as Ottawa sand: the Young's modulus of a single sand particle is $105 \mathrm{GPa}$ [40], whereas the Young's modulus of Ottawa sandstone is only $1 \mathrm{GPa}$ [41]. We perform the calibration in plane strain conditions, according to the three following steps:

1. We determine the stiffness of the inter-granular joint elements by matching the global Young's modulus of the POROFIS model with that of a reference specimen tested experimentally;

2. With the calibrated inter-granular joint stiffness, we calibrate the viscous parameters of the grain FEs so as to match the steady-state strain rate observed during secondary creep in the reference experiments; 
3. We determine the inter-granular joint strength by matching the time of tertiary creep initiation predicted with POROFIS with that measured experimentally (note: intra-granular joint parameters are discussed later in Section 4).

Table 1 summarizes the joint-enriched Finite Element parameters calibrated for the POROFIS model, and the calibration procedure is detailed below.

Table 1: Parameters calibrated for the POROFIS model with inter-granular joints only.

\begin{tabular}{cccc}
\hline \hline \multicolumn{5}{c}{ Grain } \\
\hline$E(\mathrm{GPa})$ & $\nu(-)$ & $\gamma_{0}\left(\right.$ day $\left.^{-1}\right)$ & $n(-)$ \\
\hline $4.3 \times 10^{4}$ & 0.3 & $2.0 \times 10^{-5}$ & 4.0 \\
\hline \hline \multicolumn{5}{c}{ Inter-granular Joint } \\
\hline$k_{t}(\mathrm{MPa} / \mathrm{mm})$ & $k_{n}(\mathrm{MPa} / \mathrm{mm})$ & $k_{t n}(\mathrm{MPa} / \mathrm{mm})$ & $\sigma_{R}(\mathrm{MPa})$ \\
\hline $1.0 \times 10^{4}$ & $1.0 \times 10^{5}$ & 0 & 6.13 \\
\hline$C(\mathrm{MPa})$ & $\eta($ degrees $)$ & $\beta(-)$ \\
\hline 6.13 & 30 & 1.0 \\
\hline \hline
\end{tabular}

Stiffness of inter-granular joints. In order to obtain the global stress/strain curve of the POROFIS model (as opposed to the stress/strain curve of individual FEs or joint elements), we calculate the average values of stresses and strains over the entire set of integration points. The reference Young's modulus of the polycrystal $(23 \mathrm{GPa})$ is that measured experimentally during a uniaxial compression test [39]. As shown in Figs. 7 and 8, in a log scale, the global Young's modulus calculated numerically increases almost linearly with the joint stiffness. For very high values of the joint stiffness, the numerical specimen behaves like a solid made of grains that are perfectly glued together, and the global Young's modulus calculated numerically tends towards the Young's modulus of the grain element (43 GPa). We study five ratios $k_{t} / k_{n}$ ( $k_{t}$ is the joint tangential stiffness, $k_{n}$ is the joint normal stiffness). The green dashed lines in Figs. 7 and 8 , for $k_{t}=2 \times 10^{3} \mathrm{MPa} / \mathrm{mm}$ and $k_{n}=2 \times 10^{3} \mathrm{MPa} / \mathrm{mm}$ respectively, show that the global Young's modulus is more impacted by changes of normal stiffness than by changes of tangential stiffness. Therefore we calibrate only the normal joint stiffness and assume a fixed ratio $k_{t} / k_{n}$. Because this study focuses on crack propagation induced 
by internal glide and inter-granular slip, we consider that the tangential joint stiffness is smaller than the normal joint stiffness: $k_{t}=0.1 k_{n}$. The red dashed lines in Figs. 7 and 8 represent the value of the Young's modulus of the reference specimen (23 GPa). In Fig 8, the intersection between the red line and the curve that shows the variations of the global Young's modulus for $k_{t}=0.1 k_{n}$ provides the calibrated value of the normal joint stiffness. We obtain: $k_{n}=1 \times 10^{5} \mathrm{MPa} / \mathrm{mm}$ and $k_{t}=1 \times 10^{4} \mathrm{MPa} / \mathrm{mm}$.

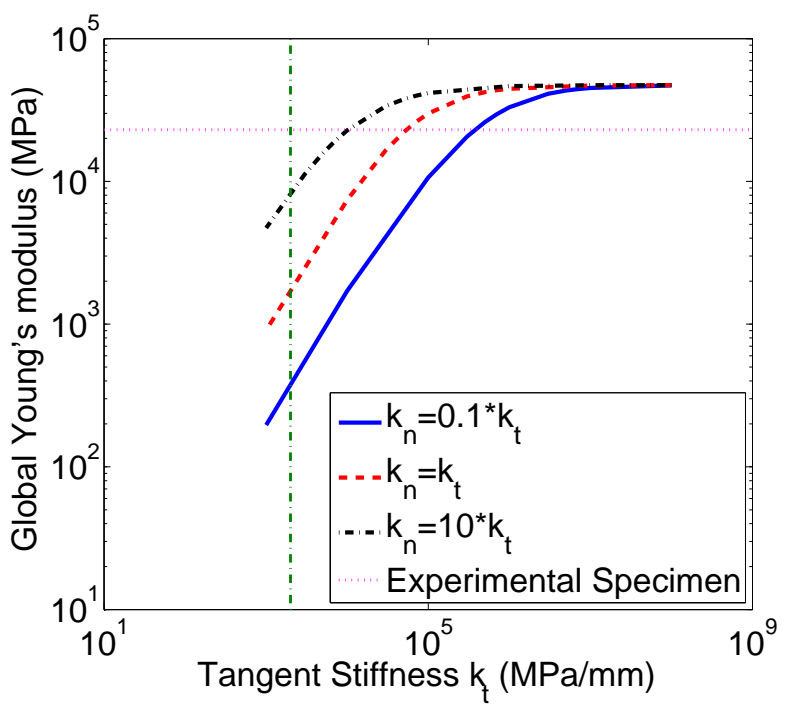

Figure 7: Evolution of the Young's modulus of the POROFIS model with inter-granular joints only, for different values of joint tangential stiffness, and for three ratios of tangential vs. normal stiffness. The vertical green dashed line shows the case $k_{t}=2 \times 10^{3} \mathrm{MPa} / \mathrm{mm}$. The horizontal red dashed line indicates the reference experimental value of the Young's modulus of the specimen (23 GPa).

Viscous parameters of the grain FEs. We obtain the viscous parameters $\left(\gamma_{0}, n\right)$ of the grain elements by matching the steady strain rate predicted by POROFIS with the strain rate predicted by the inclusion-matrix model published in [1] for a uniaxial creep tests simulated in the experimental conditions described in [39] (Fig. 9). The correlation established between the steady state creep rate and the creep loading stress made it possible to calibrate the two visco-plastic parameters. We obtained: $\gamma_{0}=2.0 \times 10^{-5} \mathrm{day}^{-1}$, $n=4.0$. We simulated two other uniaxial creep tests, performed under 2 and 


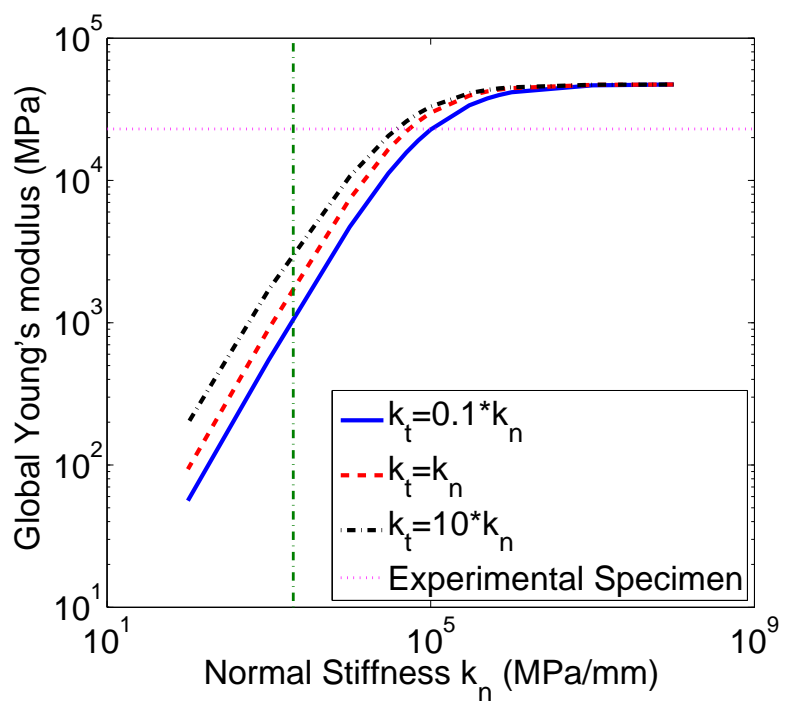

Figure 8: Evolution of the Young's modulus of the POROFIS model with inter-granular joints only, for different values of joint normal stiffness, and for three ratios of tangential vs. normal stiffness. The vertical green dashed line shows the case $k_{n}=2 \times 10^{3} \mathrm{MPa} / \mathrm{mm}$. The horizontal red dashed line indicates the reference experimental value of the Young's modulus of the specimen (23 GPa). 
$15 \mathrm{MPa}$ (black triangles in Fig. 9), and verified that the calibrated values of $\left(\gamma_{0}, n\right)$ were satisfactory.

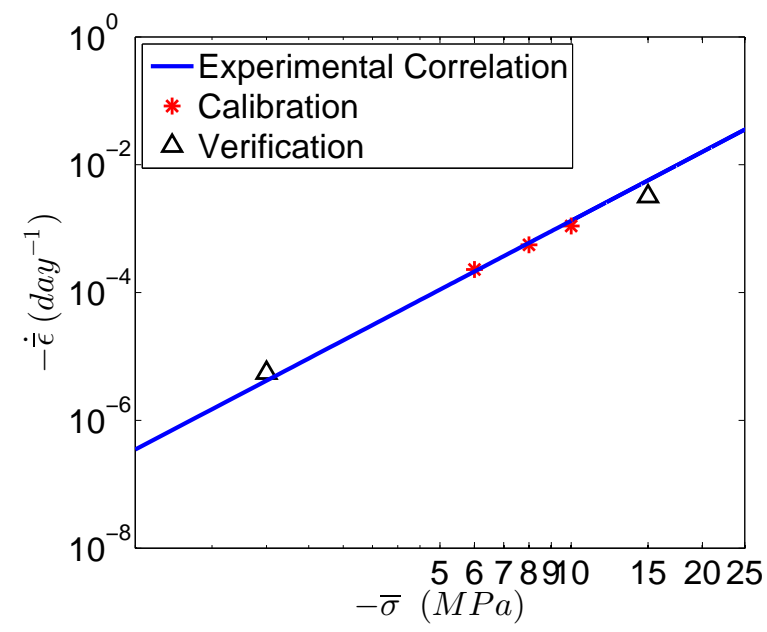

Figure 9: Correlation between macroscopic axial strain rate and macroscopic axial stress, obtained from the simulation of the uniaxial creep tests with POROFIS, in plane strain. The correlation line is obtained from a previous numerical study [1] based on experimental results reported in [39]. Red stars: numerical results used for calibration. Black triangles: numerical results used for verification.

Tensile strength of the inter-granular joint elements. As explained in Section 3.1, the joint strength criterion depends on the tensile strength parameter $\sigma_{R}$, the joint cohesion $C$, the joint friction angle $\eta$, and the ductility parameter $\beta$. At the transition between secondary and tertiary creep, the strain rate increases significantly. We expect that the initiation of tertiary creep will take more time when the joint strength, the joint cohesion, or the joint ductile parameter increases. In uniaxial creep tests performed under $30 \mathrm{MPa}$ [39], the initiation of tertiary creep occurred after 0.22 days (i.e. 5.2 hours). A good match is found for $\sigma_{R}=C=6.13 \mathrm{MPa}, \eta=30^{\circ}$, and $\beta=1$ (along with the calibrated values for joint stiffness and grain viscous parameters). We verify that the tensile strength of the joint satisfies the criterion stated above (Eq. 12). 
Fig. 10 confirms that lower strength values ( $5 \mathrm{MPa}, 6 \mathrm{MPa}$ ) lead to an early triggering of tertiary creep, while higher strength values $(7 \mathrm{MPa}, 10$ $\mathrm{MPa}$ ) lead to a later triggering of tertiary creep. It was expected that the tensile strength of the joints would be lower than that of the grains and higher than that of the polycrystal, because the joints represent defects that are naturally present in the polycrystal. We verified that the transition between secondary and tertiary creep was independent from the time increment size used in the simulations.

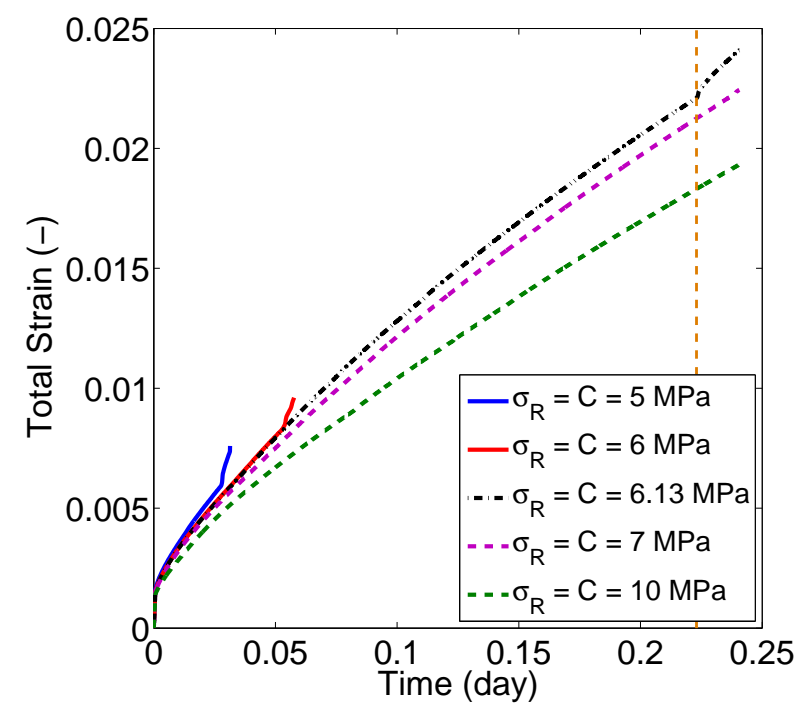

Figure 10: Calibration of the joint element strength parameters of the POROFIS model. For $\sigma_{R}=5 \mathrm{MPa}, 6 \mathrm{MPa}$, and $6.13 \mathrm{MPa}$, the creep acceleration noted at the end of the simulation corresponds to the initiation of tertiary creep. The vertical dashed line (at $\mathrm{t}=0.22$ days) indicates the initiation of tertiary creep noted during a uniaxial creep test performed under $30 \mathrm{MPa}$ [39].

\section{Numerical study of viscous damage and fatigue mechanisms in halite}

In the following, we compare the effects of various viscous damage and fatigue mechanisms that operate in halite polycrystals during uniaxial creep loading tests and cyclic triaxial tests. We use the two following models:

1. POROFIS model (1): inter-granular crack propagation accounted for by damage evolution in one family of joint elements; 3,368 surface el- 
ements and 678 inter-granular joint elements; calibrated parameters presented in Section 3.3;

2. POROFIS model (2): inter-granular and intra-granular crack propagation accounted for by damage evolution in two families of joint elements; 3,368 surface elements, 678 inter-granular joint elements and 4,294 intra-granular joint elements; calibrated parameters presented in Section 3.3, with additional intra-granular joint parameters (see below).

\subsection{Intra-granular vs. inter-granular crack propagation during a uniaxial creep test}

In the following, we present a creep test simulated in plane stress conditions under an axial loading stress of $9 \mathrm{MPa}$ with the joint-enriched FEM model (2), which accounts for both intra- and inter- granular crack propagation. Model parameters are the same as for model (1) for the grain finite elements and for the inter-granular joint elements (see Section 3.3). We calibrate the intra-granular joint stiffness so as to maintain a global Young's modulus of $23 \mathrm{GPa}$ for the polycrystal. In the absence of reference data on the tensile strength of a single crystal, we assume that the intra-granular joints have a higher strength than the inter-granular joints. For intra-granular joint elements, we choose $\sigma_{R}^{\prime}=7.495 \mathrm{MPa}$, which is the same as the grain tensile strength calibrated with the inclusion-matrix model presented in [1] (Table 3). Note that for inter-granular joint elements, we had $\sigma_{R}=6.13$ MPa.

Fig. 11 shows the vertical (i.e. axial) stress distribution in the polycrystal at the end of the creep test. Most stress concentrations are located around joints where several angular grains are in contact. As a result, damage in the joints is mainly observed at the edges of the most angular grains (Fig. 12). Note that for the particular creep test simulated with model (2), damage propagates in intra-granular joints only (i.e. we observe no damage in intergranular joints). It would be interesting to determine the tensile and shear strengths of the single crystal in order to understand in which loading conditions cracks initiate, propagate and coalesce within the grains as opposed to between the grains. 


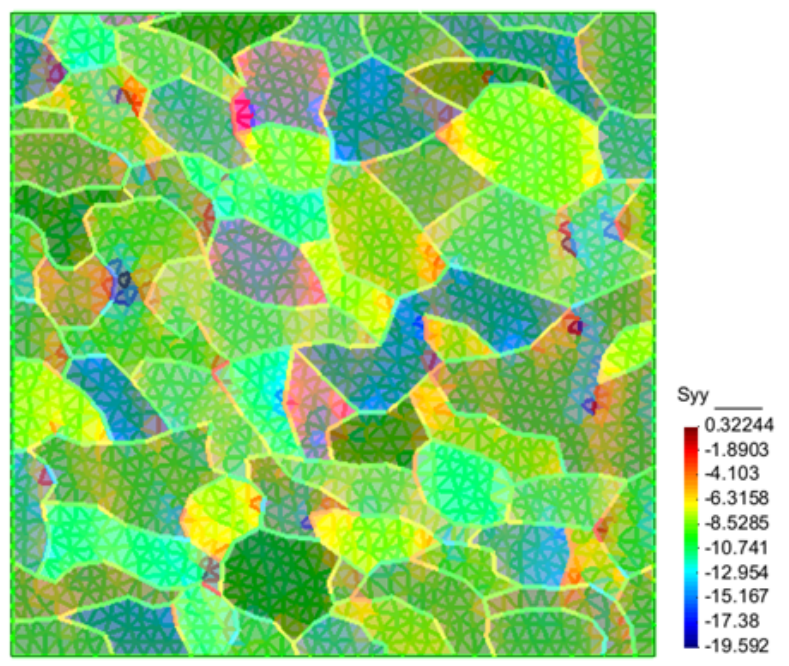

Figure 11: Vertical stress distribution $\left(S_{y y}, \mathrm{MPa}\right)$ at the end of the uniaxial creep test simulated with the joint-enriched FEM model that accounts for both intra- and intergranular crack propagation. The test duration was 0.018 day, i.e. about 26 minutes.

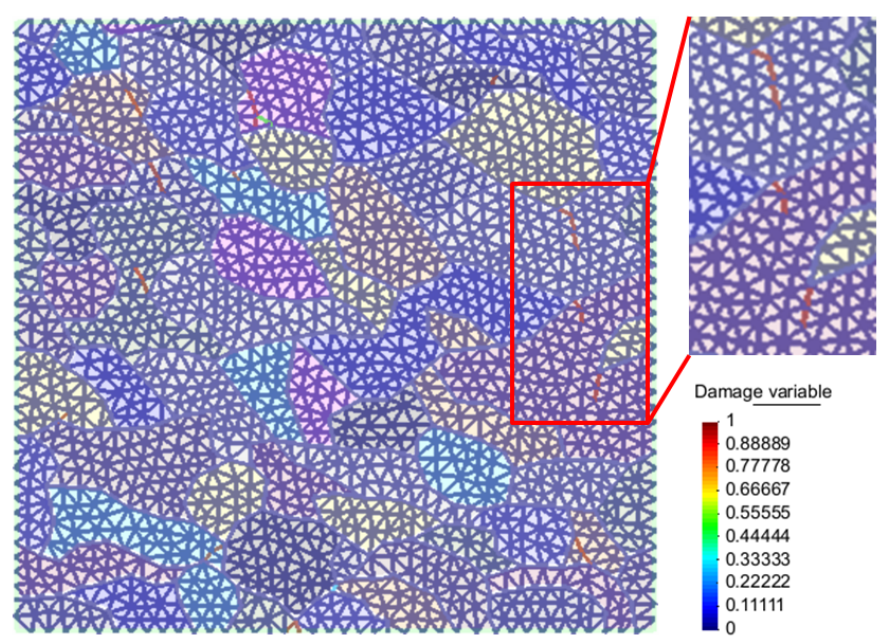

Figure 12: Distribution of damage in the joints at the end of the unaxial creep test simulated with the joint-enriched FEM model that accounts for both intra- and intergranular crack propagation. The test duration was 0.018 day, i.e. about 26 minutes.

4.2. Prediction of inter-granular crack propagation during cyclic loading tests In order to design salt caverns for natural gas storage or compressed air energy storage (CAES), it is critical to understand the evolution of the 
mechanical properties of halite subject to cyclic loading. Typically, geological storage is done under pressures ranging between $5 \mathrm{MPa}$ and $20 \mathrm{MPa}$, with loading frequencies up to one cycle per day $[42,43,44]$. Cyclic loading tests at such low frequencies are experimentally challenging, because they are time consuming and require sophisticated power supply systems. We propose to address this issue by performing a series of virtual experiments with the joint-enriched FEM model of inter-granular crack propagation (1) presented above. Simulations explained in the following are done at the laboratory scale in plane stress conditions, which is in better agreement with the stress conditions applied in typical cyclic loading tests than plane strain conditions. Table 2 summarizes the five loading tests simulated with model (1). In order to track the gradual degradation of the stiffness of the polycrystal, we simulate rapid unloading and reloading after each loading cycle. We use the slope of the corresponding stress/strain curve to calculate the Young's modulus at the peak of each cycle (see Fig. 13a and 13b).

Table 2: Cyclic loading tests performed with the joint-enriched FEM model of intergranular crack propagation (1 - axial direction; 3 - lateral direction).

\begin{tabular}{cccc}
\hline $\begin{array}{c}\text { Test } \\
\text { Number }\end{array}$ & Loading type & Applied stresses (MPa) & Period (day) \\
\hline 1 & $\begin{array}{c}\text { uniaxial } \\
\left(\sigma_{1} \geq \sigma_{3}=0\right)\end{array}$ & $\sigma_{1, \max }=5, \sigma_{1, \min }=0$ & $T=2$ \\
\hline 2 & $\begin{array}{c}\text { uniaxial } \\
\left(\sigma_{1} \geq \sigma_{3}=0\right)\end{array}$ & $\sigma_{1, \max }=10, \sigma_{1, \min }=0$ & $T=2$ \\
\hline 3 & $\begin{array}{c}\text { triaxial } \\
\left(\sigma_{1} \geq \sigma_{3}>0\right)\end{array}$ & $\sigma_{1, \max }=15, \sigma_{1, \min }=5, \sigma_{3}=5$ & $T=2$ \\
\hline 4 & $\begin{array}{c}\text { triaxial } \\
\left(\sigma_{3} \geq \sigma_{1}>0\right)\end{array}$ & $\sigma_{3, \max }=15, \sigma_{3, \min }=5, \sigma_{1}=5$ & $T=2$ \\
\hline 5 & $\begin{array}{c}\text { triaxial } \\
\left(\sigma_{3} \geq \sigma_{1}>0\right)\end{array}$ & $\sigma_{3, \max }=15, \sigma_{3, \min }=5, \sigma_{1}=5$ & $T=20$ \\
\hline
\end{tabular}

Influence of the stress amplitude (tests 1-2). The comparison of tests 1 and 2 shows that: (a) When the stress amplitude increases, the number of cycles before failure decreases (Fig. 13a-Fig. 13c). For example, the polycrystal can undergo more than 20 loading cycles before failure for a loading stress of $5 \mathrm{MPa}$ whereas it reaches failure after the $6^{\text {th }}$ cycle for a loading stress of $10 \mathrm{MPa}$ (Fig. 13b and 13d); (b) For the same number of cycles, 
strains and damage increase with stress amplitude (Fig. 13b, Fig. 13d and Fig. 14).

Influence of the confining stress (tests 1-2-3). The comparison of tests 2 and 3 shows that for the same axial stress amplitude, more confining stress delays failure (Fig. 13c - Fig. 13e) and reduces strains and damage (Fig. 13d, Fig. 13f and Fig. 14). The confining pressure effectively reduces the viscous deformation that induces fatigue in the polycrystal, which increases the strength of the polycrystal and its ability to sustain more cycles. For the same deviatoric stress, a lower axial stress delays failure (Fig. 13a - Fig. 13e) and reduces strains and damage (Fig. 13b, Fig. 13f and Fig. 14). Note that in tests 1, 2 and 3, the stress-strain cycles tend to a limit behavior, in which the same constant strain increment adds up at each cycle. Note that with the inclusion-matrix model that we presented in [1] and that we use in Section 5 for comparison, we were able to predict that the Young's modulus decreases when the number of broken grains increases [1], but we could not predict properly the progressive degradation of the Young's modulus with the number of cycles, because failure occurred shortly after the first grain breakage. By contrast, in the proposed JFEM model of intergranular crack propagation, the Young's modulus decreases with the number of loading cycles according to an exponential relationship, which is in agreement with experimental observations made in salt [39, 45], as well as in other geomaterials such as basalt [46]. 


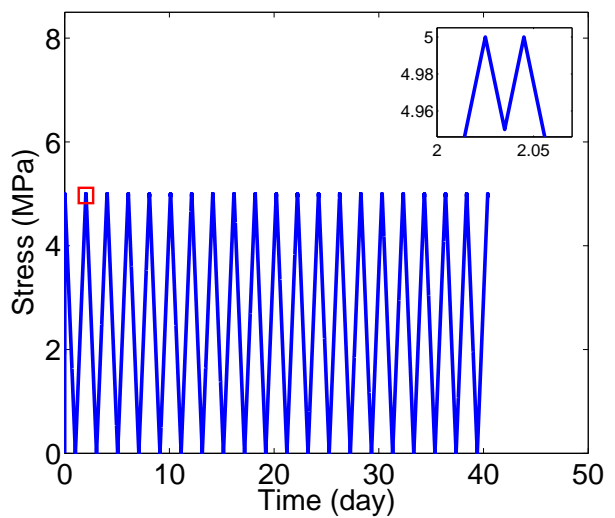

(a) Stress-time history for test 1

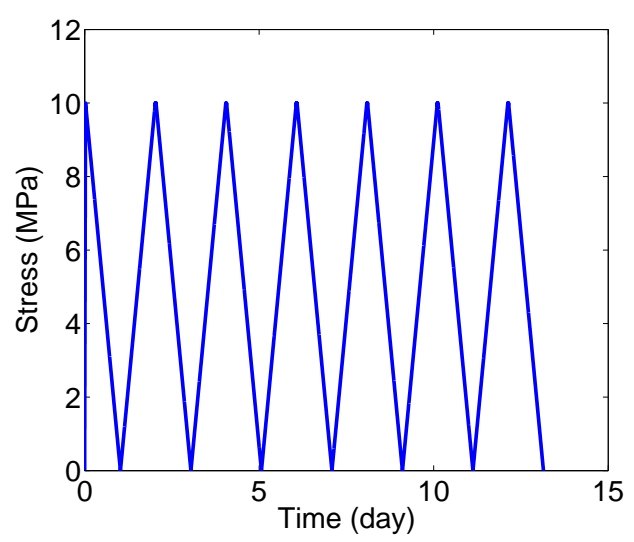

(c) Stress-time history for test 2

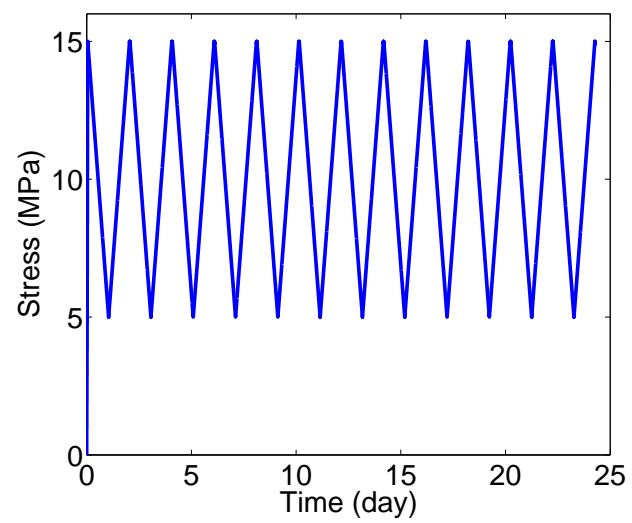

(e) Stress-time history for test 3

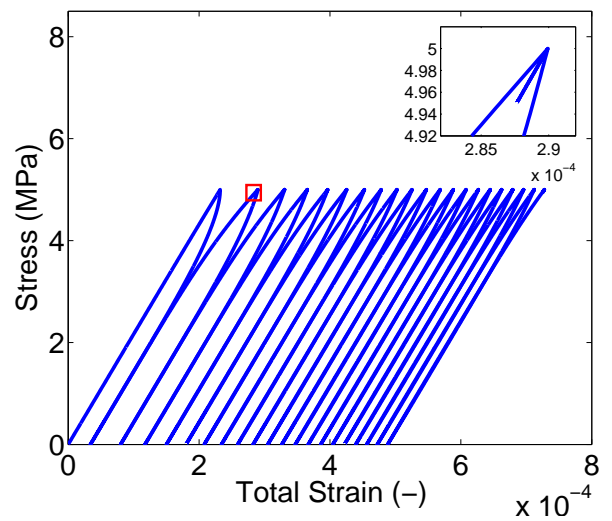

(b) Stress-strain for test 1

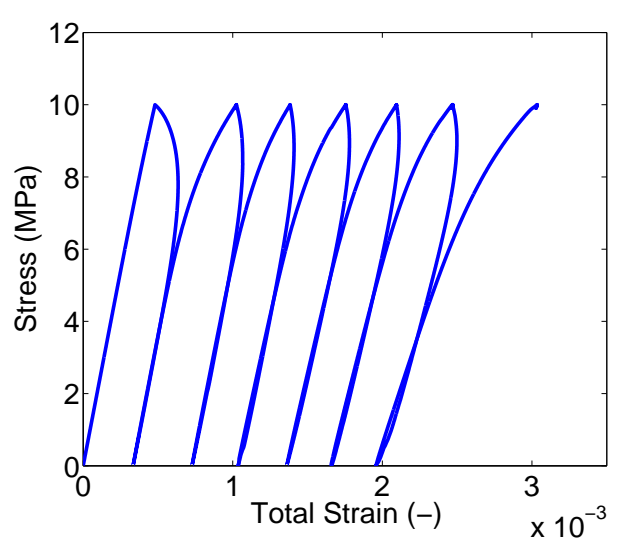

(d) Stress-strain for test 2

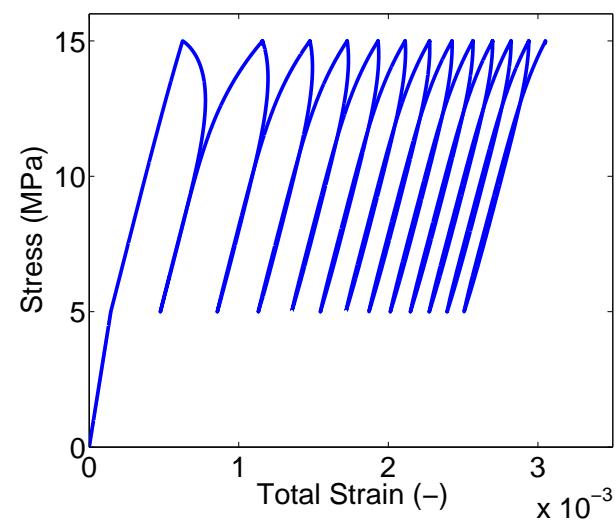

(f) Stress-strain for test 3

Figure 13: Cyclic loading tests 1-3 (verticald deviatoric stress), simulated with the jointenriched FEM model of inter-granular crack propagation. 


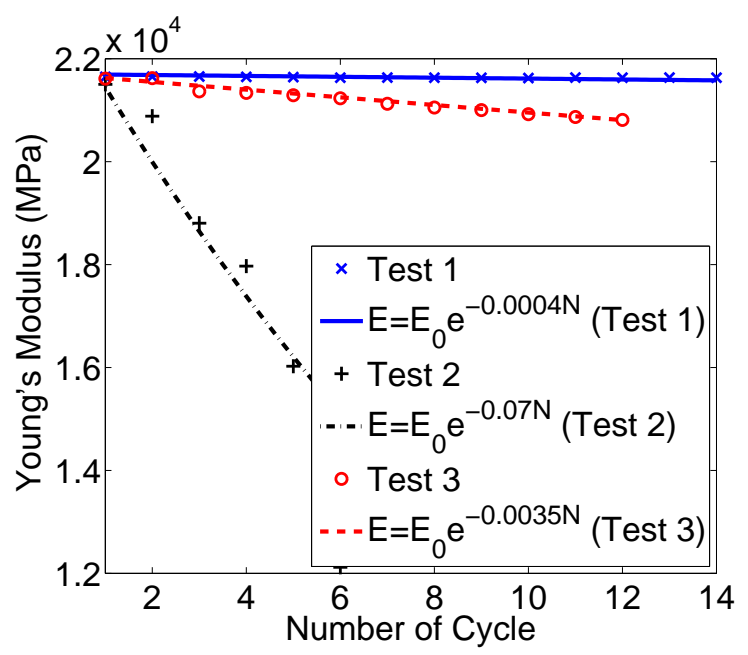

Figure 14: Variations of the polycrystal Young's modulus with the number of cycles in tests $1-3$.

Influence of the loading direction vs. polycrystal anisotropy (tests 3-4). The only difference between tests 3 and 4 is the direction of the deviatoric stress: deviatoric compression is vertical in test 3 , and horizontal in test 4. The stress-strain response is similar in both tests (Figs. 13e, 13f, 15a and $15 \mathrm{~b}$ ), which shows that the polycrystal model is relatively homogeneous (i.e. the orientations of grain FEs and joints elements approximatively follow a uniform distribution). Because the deviatoric stress is horizontal in test 4, damage after the first loading cycle mainly develops in horizontal joints (parallel to the differential compression axis - see Fig. 16).

Influence of the loading frequency (tests 4-5). The period of the loading cycles in test 5 is 10 times longer than in test 4 . Therefore in test 5 , more visco-plastic deformation accumulates at each cycle, which results in earlier failure (Fig. 15a - Fig. 15c), higher strains (Fig. 15b - Fig. 15d) and higher damage (Fig. 17). Like in tests 1-3, the evolution of the Young's modulus with the number of cycles follows an exponential trend (note that in tests 1-3, the Young's modulus was calculated from the slope of the axial stress/strain curves, whereas in tests 4-5, the Young's modulus was calculated from the slope of the lateral stress/strain curves). 


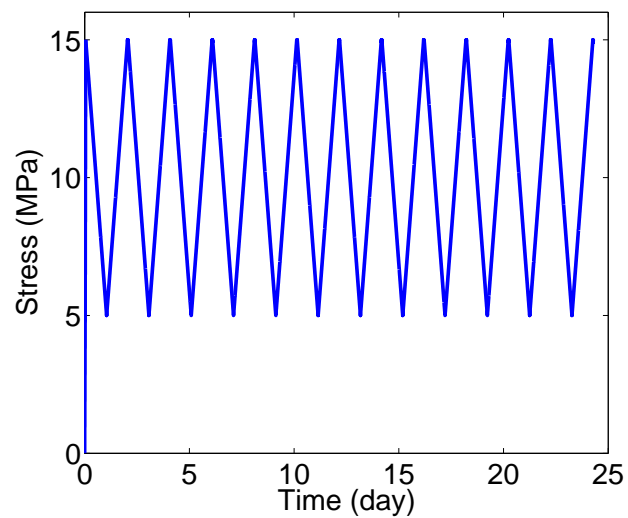

(a)

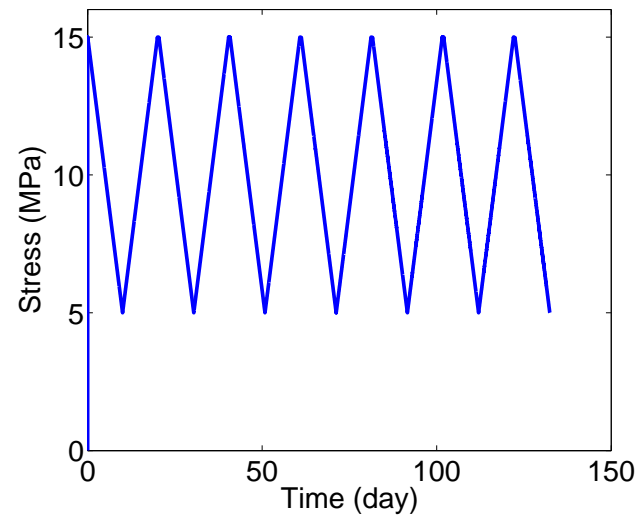

(c)

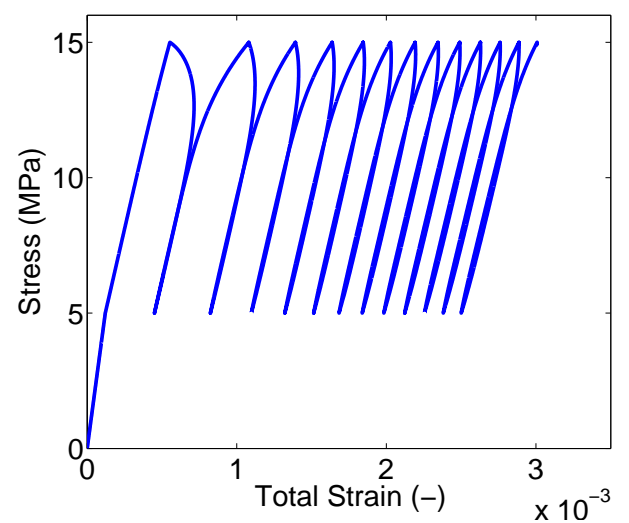

(b)

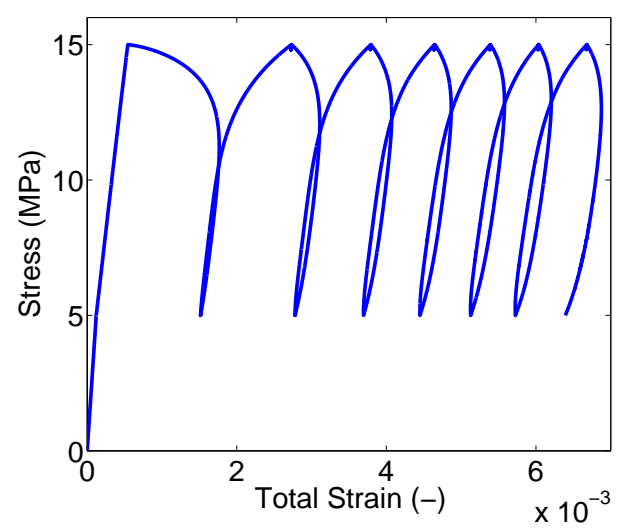

(d)

Figure 15: Cyclic loading tests 4-5 (horizontal deviatoric stress), simulated with the jointenriched FEM model of inter-granular crack propagation. 


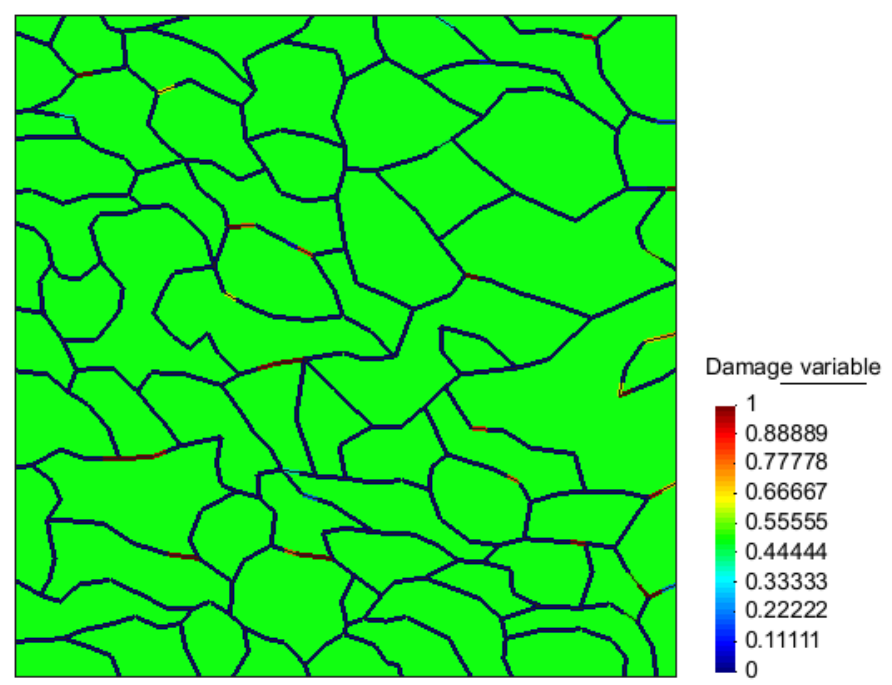

Figure 16: Damage in the joint elements at the end of the initial loading cycle in test 4 (mostly horizontal cracks).

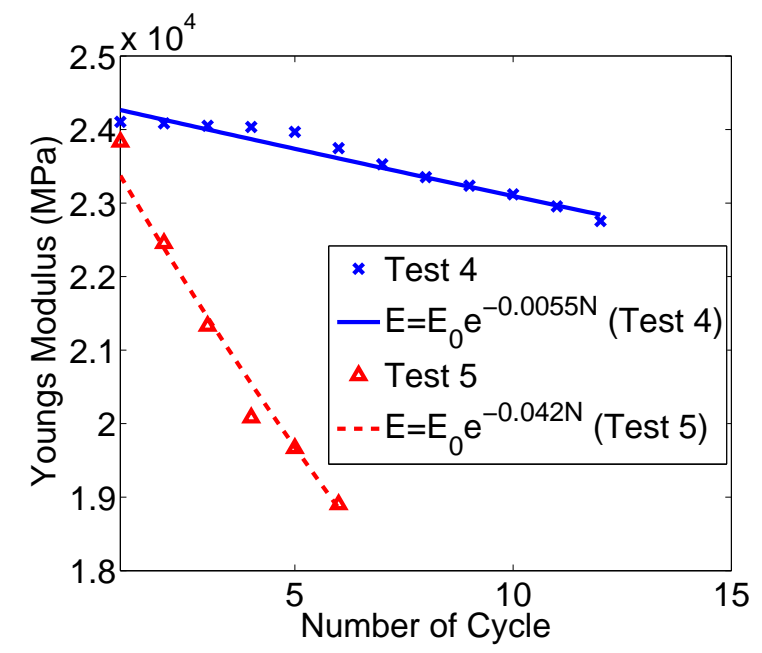

Figure 17: Variations of the polycrystal Young's modulus with the number of cycles in tests 4-5. 


\section{Comparison of the JFEM with an inclusion-matrix model}

\subsection{Hill's Tensor in 2D}

In previous work, we presented an inclusion-matrix model of grain breakage [1], based on a 3D self-consistent method. For further comparison with our 2D JFEM model, we adapt our homogenization scheme to 2D plane strain conditions. We used Hill's incremental inclusion-matrix model [47] to build up our self-consistent homogenization method (Fig. 18a and Fig. 18b). Note that other homogenization schemes upscale explicitly the yield criteria and the flow rules. For instance, the transformation field analysis deals with thermomechanical loading problems in inelastic heterogeneous media and composite materials [48]. Fritsch et al. [49] proposed a multiscale micromechanics model for the upscaling of elastoplastic properties. Nonlinear homogenization models were employed to investigate the mechanical behavior of metal-ceramic composites [50]. All of these sophisticated upscaling schemes were applied to the study of elastic-plastic or visco-plastic behavior. By contrast, our simplified inclusion-matrix model is designed to predict damage or fatigue. We considered spherical inclusions. Homogenization schemes were developed with more sophisticated shapes such as ellipsoidal, needle-shaped, and disk-shaped inclusions [51, 52]. However, in contrast to the highly anisotropic texture of laminated metals (i.e., elongated grain shapes), no significant salt grain anisotropy has ever been evidenced by experimental microstructure observations or mechanical tests. This difference may be due to the genesis mechanism of the crystals involved in salt microstructure. These crystals grow in supersaturated brine, as water evaporates. This process does not result in preferential growth directions.

In Hill's model, small variations of the local stress in the inclusions $(\boldsymbol{\sigma})$ and small variations of the far-field stress in the matrix $(\overline{\boldsymbol{\sigma}})$ are coupled to those of the microscopic strain $(\varepsilon)$ and those of the macroscopic strain $(\bar{\varepsilon})$ by the following relationship:

$$
\delta \boldsymbol{\sigma}-\delta \overline{\boldsymbol{\sigma}}=-\boldsymbol{L}^{*}:(\delta \boldsymbol{\varepsilon}-\delta \overline{\boldsymbol{\varepsilon}})
$$

in which $\boldsymbol{L}^{*}$ is Hill's tensor. The Hill's tensor for a spherical inclusion embedded in a 3D isotropic elastic matrix is available in the literature (e.g., [47]). To express Hill's tensor for a 2D plane strain case, we consider the spherical inclusion as a cylinder of circular section ("borehole") subject to internal pressure and far-field stresses (Fig. 18c). The solution is obtained by superimposing three independent displacement fields that correspond to 
three independent stress fields in plane strain conditions: (1) Displacements induced by an isotropic pressure field applied at the borehole wall; (2) Pure shear displacements; (3) Homogeneous displacements. The detailed derivations are provided in Appendix A. The Hill's tensor $\boldsymbol{L}^{*}$ for 2D plane strain conditions is finally expressed as:

$$
L_{i j k l}^{*}=\frac{E}{2(1+\nu)(3-4 \nu)}\left[\frac{1-2 \nu}{\nu} \delta_{i j} \delta_{k l}+\left(\delta_{i k} \delta_{j l}+\delta_{i l} \delta_{j k}\right)\right]
$$

in which $E$ and $\nu$ are the Young's modulus and Poisson's ratio of the matrix, and $\boldsymbol{\delta}$ is the 2D identity tensor. We verified the solution for the asymptotic case in which an internal pressure $\sigma_{p}$ is applied on the inner wall of a borehole that has an outer radius that tends to infinity.
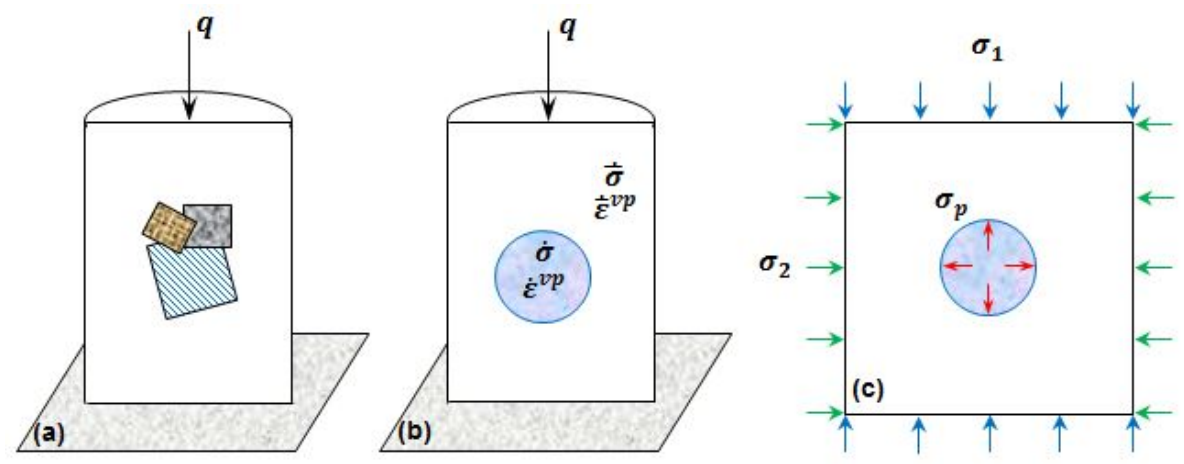

Figure 18: Cross-sectional view of the Representative Elementary Volume (REV) of salt rock considered in the homogenization scheme. (a) Salt polycrystal; (b) Inclusion-matrix model; (c) Equivalence of the grain-matrix interaction problem with that of a borehole subject to internal pressure and far-field stresses.

\subsection{Damage accommodation}

A proper account for local strain heterogeneity in polycrystalline materials would require accounting for sophisticated interaction laws between the inclusions and the matrix, such as a cohesive law to model debonding at the inclusion/matrix interface and/or a model that couples viscoplastic accommodation to damage accommodation. For instance, a second-order homogenization scheme was used for modeling the matrix behavior and stress/strain field fluctuations in cubic and hexagonal viscoplastic polycrystals and further extended to simulate texture evolution in halite polycrystals [53, 54]. Here we focus on the modeling of viscous cracking and cyclic fatigue of salt 
polycrystals. We compare the JFEM with the inclusion-matrix model presented in [1], which accounts for damage accommodation but not viscoplastic accommodation. We assume that grains break when the maximum principal microstress $\left(\sigma_{p}\right)$ exceeds the grain tensile strength $\left(\sigma_{T}\right)$. More sophisticated models of progressive grain damage could be used to account for the anisotropy induced by diffuse crack propagation in the polycrystal. For instance, fast Fourier transforms were applied to compute the growth of intergranular voids in porous polycrystalline materials, but the approach was limited to the prediction of ductile damage evolution and does not account for void nucleation induced by microstructural effects [55]. To our best knowledge, homogenization schemes proposed so far (e.g., $[56,57])$ resort to the Hill's tensor expressed for an isotropic matrix. In the self-consistent method adopted in this study, for anisotropic loading conditions and anisotropic crystal orientation distributions, modeling the progressive damage within grains would require expressing the Hill's tensor for spherical inclusions embedded in an anisotropic matrix. The required theoretical developments are beyond the scope of this paper, which focuses on the multi-scale numerical modeling of tertiary creep and viscous fatigue.

We consider a representative polycrystal that contains grains with 200 possible orientations, which are generated automatically by varying the $\theta$ angle uniformly within the interval $\left[\cos (\theta=0)=0, \cos \left(\theta=\frac{\pi}{2}\right)=1\right]$ (see Subsection 3.2). Note that the two other Euler angles $\Phi$ and $\Psi$ are zero because we focus on plane strain conditions. At each computing iteration, grains are sorted into three categories of inclusion: (1) Non-broken grain inclusion with the visco-plastic behavior described in Section 2 if $\sigma_{p}<\sigma_{T}$; (2) Breaking grain inclusion if $\sigma_{p} \geq \sigma_{T}$ during the current iteration; (3) Broken grain inclusion modeled as a void in all other cases. The stress and strain increments induced by the imposed macroscopic stress variation are derived for these three categories. The algorithm allows computing the micro-stress redistribution due to viscoplasticity, from which it is possible to update micro-strains and macro-strains. In the inclusion-matrix model, the damage variable $\left(D_{I M}\right)$ is defined as the ratio between the number of broken grains $\left(N_{b}\right)$ and the number of total grains $(N)$ in the representative polycrystal. This damage variable is used to relate the damaged macroscopic properties of the polycrystal with their initial undamaged values. 


\subsection{Self-consistent model calibration}

In our previous work [1], we calibrated the 3D inclusion-matrix model against experimental creep curves reported in the literature. We used a genetic algorithm to generate test values for the viscous parameters $\gamma_{0}$ and $n$ that are involved in the irreversible deformation law (Eq. 4). We optimized the set of $\gamma_{0}$ and $n$ values with a least square method so as to match the asymptotic strain rates at the end of the experimental creep tests. We used the same optimal viscous parameters in this plane strain study: $\gamma_{0}=5.17 \times$ $10^{-4}$ day $^{-1}$, and $n=3.58$. Note that these parameters were calibrated from secondary creep curves, before damage initiates in the polycrystal (i.e., in steady state). In our previous study, we were considering that the grain tensile strength was equal to the tensile strength of the polycrystal (2 MPa). However, it is well-known that a single grain is usually stronger than the polycrystal [38]. In order to better predict grain breakage, we calibrated the strength of the single crystal so as to match the time necessary to trigger the first grain breakage with the time that marks the transition between secondary and tertiary creep in the experiments reported in [39]. We found a tensile strength of $7.495 \mathrm{MPa}$, which is larger than the tensile strength of the polycrystal. All calibrated parameters are summarized in Table 3.

Table 3: Parameters calibrated for the inclusion-matrix model

\begin{tabular}{ccccc}
\hline \multicolumn{5}{c}{ Grain } \\
\hline$E(\mathrm{GPa})$ & $\nu(-)$ & $\gamma_{0}\left(\right.$ day $\left.^{-1}\right)$ & $n(-)$ & $\sigma_{T}(\mathrm{MPa})$ \\
\hline $2.3 \times 10^{4}$ & 0.3 & $5.17 \times 10^{-4}$ & 3.58 & 7.495 \\
\hline
\end{tabular}

5.4. Simulation of uniaxial creep tests: grain breakage vs. inter-granular crack propagation

We simulated a uniaxial creep test with the inter-granular crack propagation Finite Element model (1) and with the matrix-inclusion model of grain breakage (with 200 possible grain orientations). We used plane strain conditions in the POROFIS model (1), in order to be consistent with the formulation of the homogenization model. The axial stress was $35 \mathrm{MPa}$, which corresponds to the maximum stress amplitude that can be applied at the wall of a salt cavern by depressurization after sealing and abandonment 
[58]. The time evolution of the axial stress applied during the simulations is shown in Fig. 19.

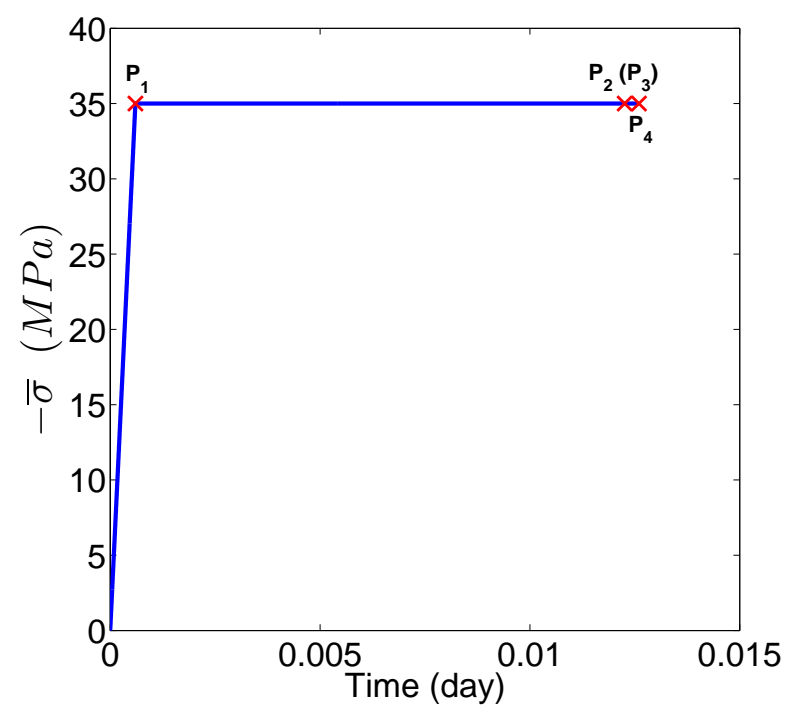

Figure 19: Stress-time input for the axial creep test simulated with the JFEM and the inclusion-matrix model. $P_{1}$ indicates the time needed to apply the total loading stress, which is increased incrementally up to $35 \mathrm{MPa} . P_{2}$ (respectively $P_{3}$ ) marks the time just before (respectively after) the transition between secondary and tertiary creep. $P_{4}$ indicates the end of the creep test.

Contrary to the inclusion-matrix model, the joint-enriched Finite Element model accounts for the geometric incompatibilities induced by shear deformation in the grains and by sliding relative displacements along grain boundaries. As expected, the macroscopic deformation of the polycrystal modeled with Finite Elements is higher than that of the polycrystal modeled with the self-consistent method (Fig. 20). For instance, the axial deformation predicted by the JFEM at the end of the creep test is twice higher than that predicted with the inclusion-matrix model. The strain-time history also indicates that the inclusion-matrix model predicts ultimate failure at the end of the test (after 0.012 days, which is about 17 minutes), whereas the JFEM model predicts that the polycrystal is still in steady state at the end of the creep test. Such discrepancies were expected, because Hill's incremental method ignores viscous accommodation. As mentioned earlier, other approaches, such as transformation field analysis, multiscale micromechanics models and nonlinear homogenization models are expected to provide a 
better match with the FE results, but they do not take damage effects into account.

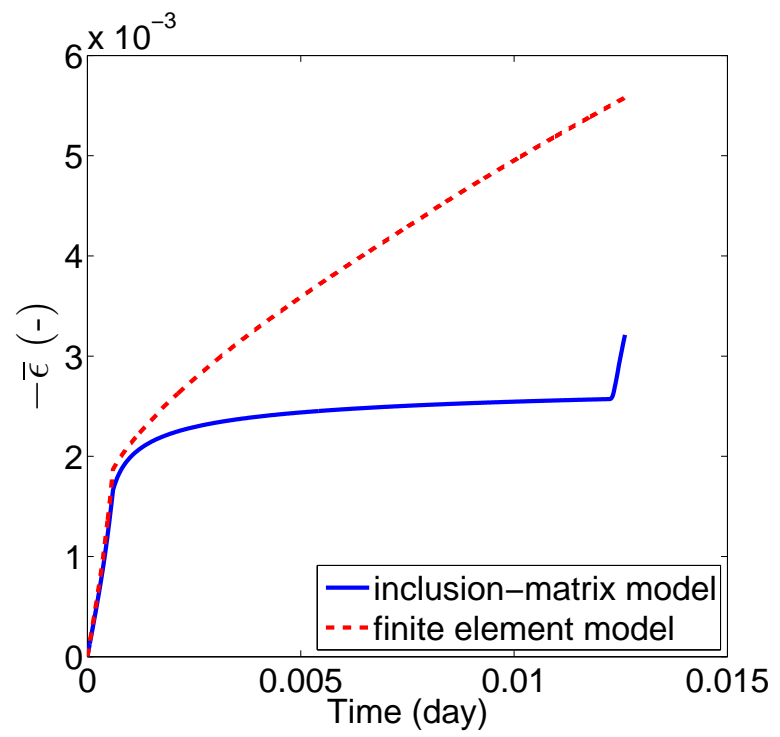

Figure 20: Time evolution of the macroscopic axial deformation of the polycrystal during the uniaxial creep test simulated with the joint-enriched FEM model of inter-granular crack propagation and with the inclusion-matrix model of grain breakage.

In both the JFEM and the inclusion-matrix models, the viscous grain parameters were calibrated so as to match secondary creep strain rates measured experimentally. In the inclusion-matrix model, the initiation of tertiary creep coincides with the first grain breakage that occurs in the polycrystal $[1,6]$. In the JFEM, joint strength was calibrated so as to capture the initiation of tertiary creep at the scale of the polycrystal. Therefore damage can propagate in the inter-granular joints before the initiation of tertiary creep, which corresponds to the coalescence of inter-granular cracks. In the present case, inter-granular cracks propagate during the incremental loading phase, before the creep stress loading is fully applied (see Fig. 24b). As a result, the stiffness of the polycrystal predicted with the JFEM is less than the stiffness of the (intact) polycrystal modeled with the self-consistent method. This is the reason why the slope of the stress/strain curve during the initial loading phase is smaller with the JFEM than with the inclusion-matrix model, as can be seen in Fig. 21.

The distributions of major and minor microscopic stresses in the inclusion- 


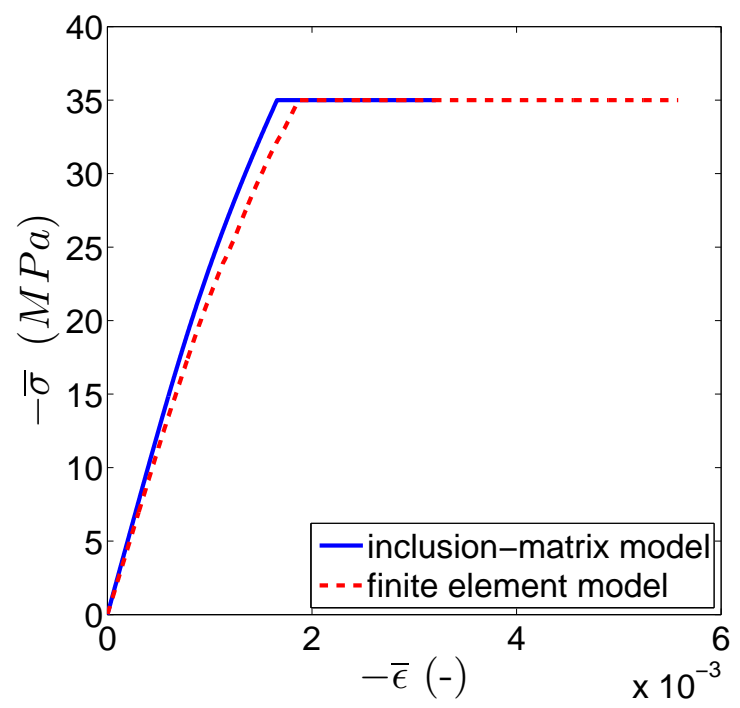

Figure 21: Macroscopic stress/strain curve of the polycrystal during the uniaxial creep test simulated with the joint-enriched FEM model of inter-granular crack propagation and with the inclusion-matrix model of grain breakage.

matrix model are shown in Figs. 22 and 23, respectively. Each point in the figure represents a principal stress value for one of the 200 grain orientations considered in the polycrystal. At stage $P_{1}$, the polycrystal is subjected to the total macroscopic stress load (35 MPa), but viscoplastic deformation in the grains has not started. At stage $P_{2}$, viscous shear deformation in the grains induce stress concentrations within the polycrystal, which translates into higher microscopic stresses (more compression, and more tension). Microscopic slip mechanisms result in a redistribution of microscopic stresses. In particular, tensile microscopic stresses increase continuously in the lateral directions until the strength threshold is reached (Fig. 22). The maximum tensile micro-stress noted in the polycrystal is $7.495 \mathrm{MPa}$, which corresponds to the grain tensile strength. At stage $P_{3}$, the tensile stress in some grains exceeds the grain tensile strength, which results into local grain breakage (i.e., zero micro-stress in Fig. 23) and global stress redistribution. To restore the stress balance, non-broken grains become subjected to larger compressive stress in both axial and lateral directions (Figs. 22 and 23).

Post-processed Finite Element results explain the differences between the two models noted in Figs. 20 and 21. In the JFEM model, concentrations of 


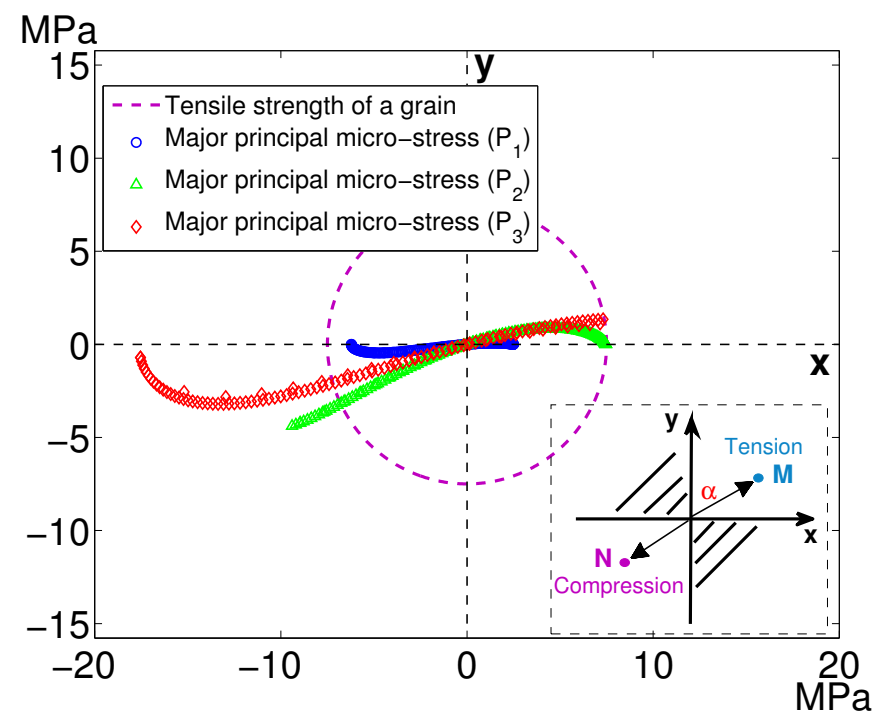

Figure 22: Distribution of the major principal micro-stresses of the polycrystal during the creep test simulated with the inclusion-matrix model. Each dot represents one grain orientation. $P_{1}$ marks the state of the polycrystal after completion of the loading; $P_{2}$ (respectively $P_{3}$ ) marks the state of the polycrystal just before (respectively after) the transition between secondary and tertiary creep. 


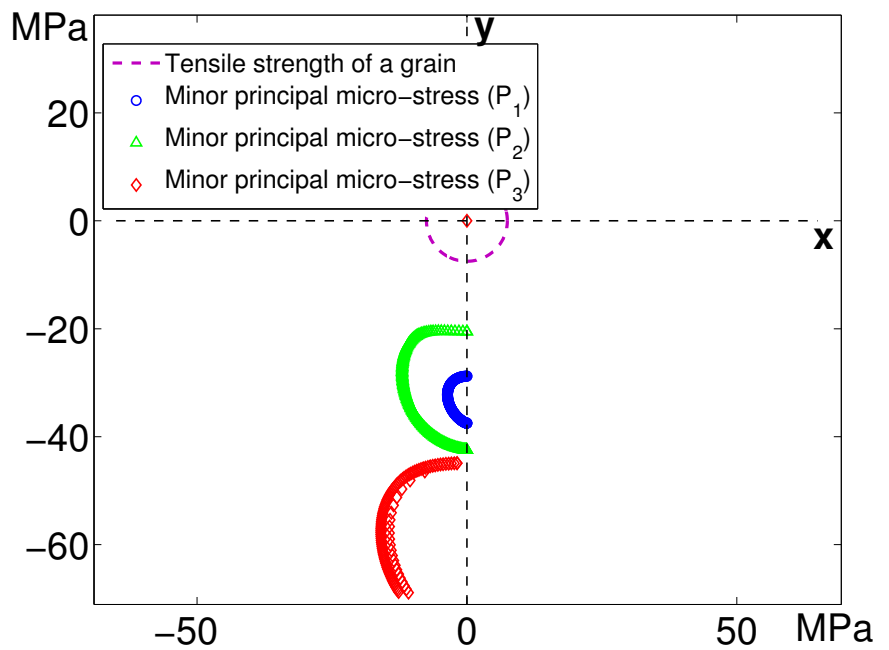

Figure 23: Distribution of the minor principal micro-stresses of the polycrystal during the creep test simulated with the inclusion-matrix model. Each dot represents one grain orientation. $P_{1}$ marks the state of the polycrystal after completion of the loading; $P_{2}$ (respectively $P_{3}$ ) marks the state of the polycrystal just before (respectively after) the transition between secondary and tertiary creep. 
vertical stress occur at the contact of angular grains even before the initiation of creep (Fig. 24a), which results in inter-granular slip and subsequent damage in some joints that are parallel to the loading axis (Fig. 24b). Fig. 25a and Fig. 25b show that the orientations of the nodal principal stresses follow a distribution similar to that obtained with the inclusion-matrix model: local major principal stresses are tensile and compressive stresses oriented normal to the loading axis, and local minor stresses are compressive stresses oriented parallel to the loading axis. Local stress magnitudes are higher than those obtained with the inclusion-matrix model, because the JFEM accounts for geometric incompatibilities, and the inclusion-matrix model does not. At the end of the creep test, viscous shear deformation in the grains induces more geometric incompatibilities, which results in higher stress (Fig. 26a) and inter-granular crack coalescence (Fig. 26b). Nodal principal stresses also increase during the creep process. However, the distribution of stress orientations remains the same, as can be seen in Fig. 27a and Fig. 27b. In the inclusion-matrix model, geometric incompatibilities are not accounted for, and ultimate failure occurs shortly after the first grain breakage. We conclude that the joint-enriched FEM model of inter-granular crack propagation provides a more realistic representation of the microstructure evolution and of the creep behavior of halite than the inclusion-matrix model. The main advantage of the inclusion-matrix model is that it is less computation-intensive than a detailed Finite Element model like the JFEM presented above.

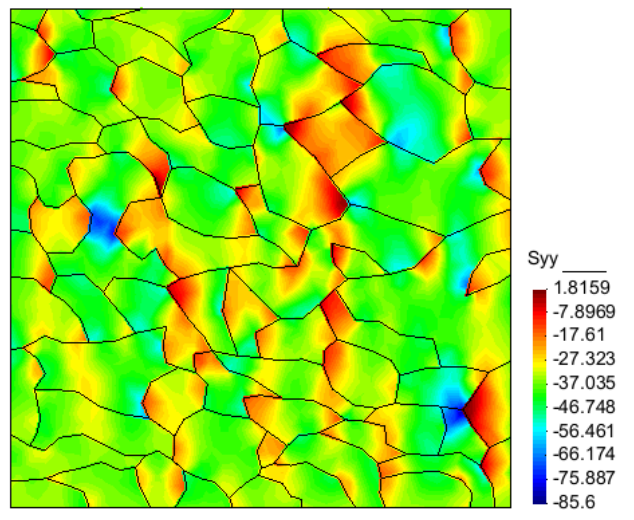

(a) Vertical stress Syy (in MPa)

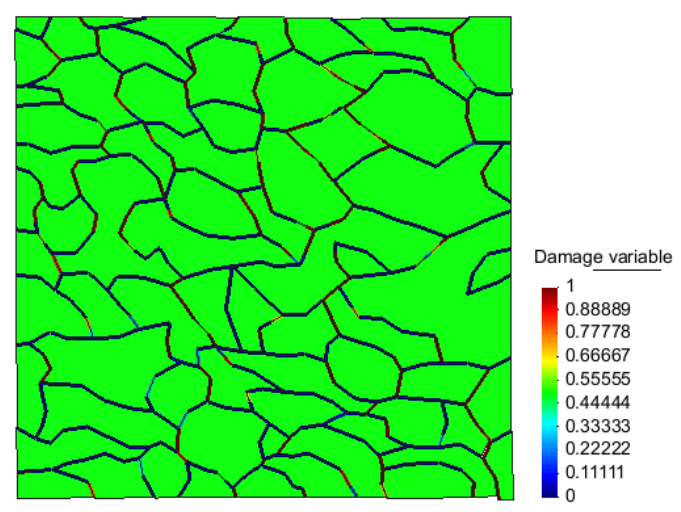

(b) Damage in the joint elements - Deformed mesh $(10 \mathrm{x})$

Figure 24: Results of the creep test obtained with the joint-enriched FEM inter-granular crack propagation model after the initial loading phase $\left(P_{1}\right)$. 


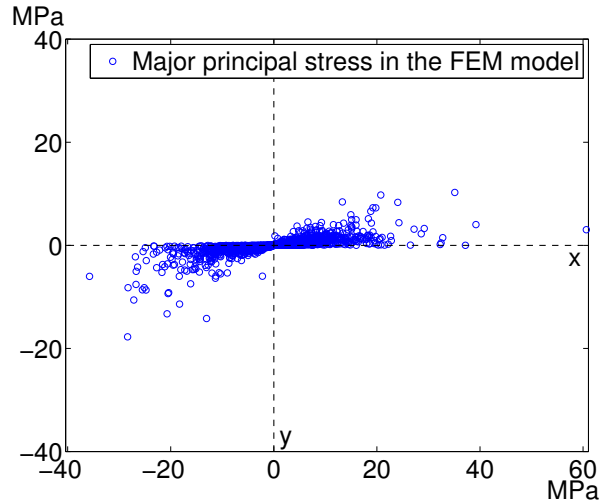

(a) Major principal stress (in MPa)

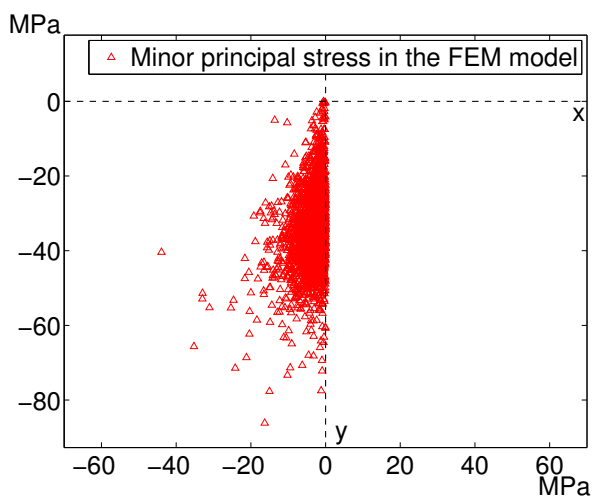

(b) Minor principal stress (in MPa)

Figure 25: Nodal principal stresses obtained with the joint-enriched FEM inter-granular crack propagation model after the initial loading phase $\left(P_{1}\right)$.

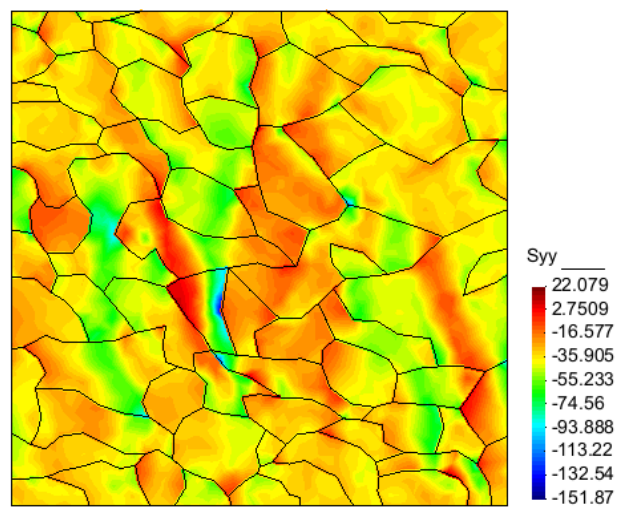

(a) Vertical stress Syy (in MPa)

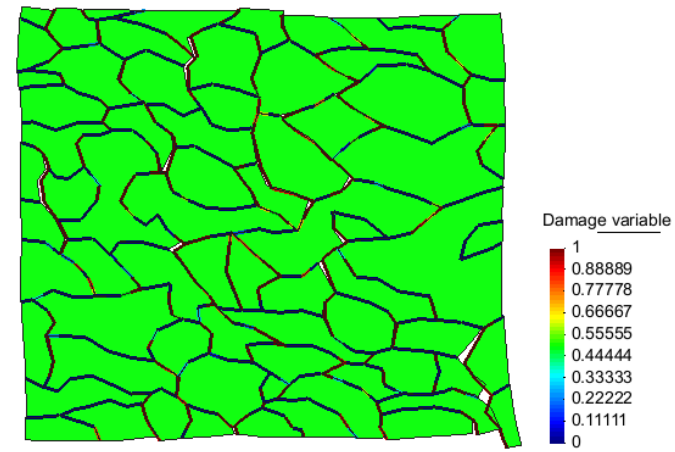

(b) Damage in the joint elements - Deformed mesh $(10 \mathrm{x})$

Figure 26: Results of the creep tests obtained with the joint-enriched FEM inter-granular crack propagation model at the end of the creep test $\left(P_{4}\right)$. 


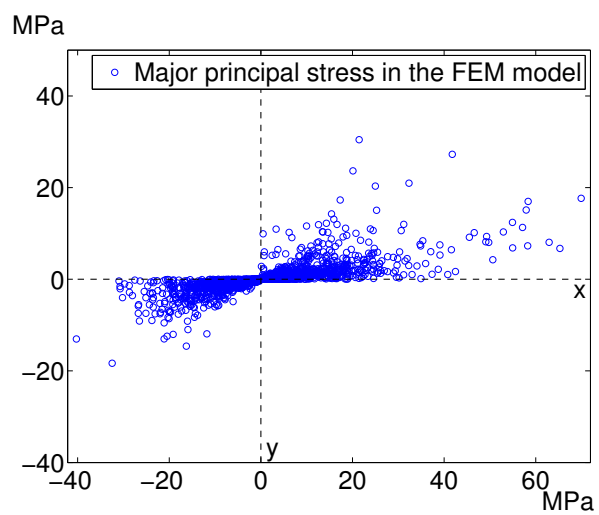

(a) Major principal stress (in MPa)

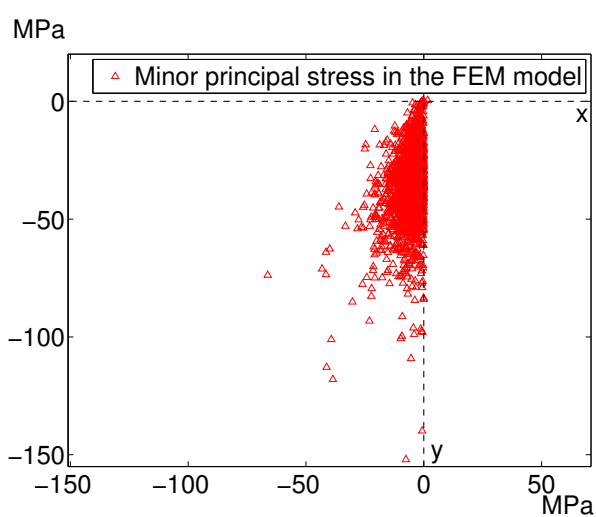

(b) Minor principal stress (in MPa)

Figure 27: Nodal principal stresses obtained with the joint-enriched FEM inter-granular crack propagation model at the end of the creep test $\left(P_{4}\right)$.

\section{Conclusions}

In this paper, we present a new Joint-enriched Finite Element Method (JFEM) to predict viscous damage and fatigue in halite polycrystals in 2D. Different visco-plastic finite elements are used to represent grains of different orientations, and joint elements are used for modeling crack propagation. The mesh is generated from an EBSD map of salt microstructure. Simulations of uniaxial creep tests show that, as it could be expected theoretically, viscous shear deformation in grains causes geometric incompatibilities. Numerical results also show that the transition between secondary and tertiary creep corresponds to inter-granular crack coalescence. The JFEM model captures the mechanical behavior of halite under cyclic loading, mainly: (a) Higher stress amplitude, lower confining stress, and lower loading frequency increase the deformation and damage of the polycrystal, which results in earlier failure; (b) The Young's modulus of the polycrystal decreases exponentially with the number of cycles, which is in agreement with experimental observations; (c) The FEM polycrystal presents similar behaviors for cyclic tests of different deviatoric loading direction, which confirms the relative homogeneity of the polycrystals modeled with the joint-enriched FEM. Simulations with intra- and inter- granular joint elements show that most stress concentrations occur in intra-granular joints where several angular grains are in contact.

Results of creep tests obtained with the JFEM are compared to those obtained with an inclusion-matrix model that accounts for damage accom- 
modation due to grain breakage, in which grains are considered as spherical inclusions of different orientations embedded in an infinite isotropic matrix. Both the JFEM and inclusion-matrix models are calibrated against experimental creep tests to produce a Young's modulus of $23 \mathrm{GPa}$ for the polycrystal, to match secondary creep strain rates and to match the time of tertiary creep initiation. The inclusion-matrix model is less computation-intensive than the JFEM model, but the JFEM model provides a more realistic interpretation of microstructure evolution. In the inclusion-matrix model, the absence of grain geometric rearrangement over time is translated into a brutal failure that occurs shortly after the first grain breakage that triggers tertiary creep. Moreover, the JFEM model highlights the evolution of microstresses and microcracks upon viscous deformation.

In order to gain fundamental understanding of anisotropic damage mechanisms in halite, we are currently developing an inclusion-matrix model that accounts for damage anisotropy in grains embedded in an anisotropic matrix. Future joint-enriched FEM modeling will focus on the representation of pores and cracks in the polycrystal. The JFEM is of great promise to understand complex phenomena of viscous accommodation coupled with grain interface debonding.

\section{Acknowledgements}

Financial support for this research was provided by the National Science Foundation (Grant No. CMMI-1362004/1361996).

\section{Appendix A. 2D plane strain Hill's tensor}

As a result of an internal stress inside the inclusion (Fig. 18(c)), the displacement $u^{1}$ and stress fields $\sigma^{1}$ are

$$
\begin{gathered}
u_{r}^{1}=a_{1} \frac{R^{2}}{r}, \quad u_{\theta}^{1}=0, \\
\sigma_{r r}^{1}=-a_{1} \frac{E}{1+\nu} \frac{R^{2}}{r^{2}}, \quad \sigma_{\theta \theta}^{1}=a_{1} \frac{E}{1+\nu} \frac{R^{2}}{r^{2}},
\end{gathered}
$$

in which $R$ is the radius of the spherical inclusion, $a$ is a stress-dependent

coefficient, $E$ is the Young's modulus, and $\nu$ is the Poisson's ratio. 
For a pure shear displacement field homogeneous in $r^{-3}$, the displacement $u^{2}$ and stress fields $\sigma^{2}$ are

$$
\begin{gathered}
u_{r}^{2}=a_{2} \frac{R^{4}}{r^{3}} \cos 2 \theta, \quad u_{\theta}^{2}=a_{2} \frac{R^{4}}{r^{3}} \sin 2 \theta, \\
\sigma_{r r}^{2}=-a_{2} \frac{3 E}{1+\nu} \frac{R^{4}}{r^{4}} \cos 2 \theta, \quad \sigma_{\theta \theta}^{2}=a_{2} \frac{3 E}{1+\nu} \frac{R^{4}}{r^{4}} \cos 2 \theta, \quad \sigma_{r \theta}^{2}=-a_{2} \frac{3 E}{1+\nu} \frac{R^{4}}{r^{4}} \sin 2 \theta .
\end{gathered}
$$

For an auxiliary homogeneous displacement field, the displacement $u^{3}$ and stress fields $\sigma^{3}$ are

$$
\begin{gathered}
u_{r}^{3}=2 a_{3}(1-\nu) \frac{R^{2}}{r} \cos 2 \theta, \quad u_{\theta}^{3}=-a_{3}(1-2 \nu) \frac{R^{2}}{r} \sin 2 \theta, \\
\sigma_{r r}^{3}=-a_{3} \frac{2 E}{1+\nu} \frac{R^{2}}{r^{2}} \cos 2 \theta, \quad \sigma_{\theta \theta}^{3}=0, \quad \sigma_{r \theta}^{2}=-a_{3} \frac{E}{1+\nu} \frac{R^{2}}{r^{2}} \sin 2 \theta .
\end{gathered}
$$

The 2D Hill's tensor $L^{*}$, correlated by a stress tensor $A$ and a strain tensor $B$, has to satisfy the condition that when $r=R$, we have

$$
\begin{gathered}
\sigma \cdot n=A \cdot n, \\
u=B \cdot x, \\
B=-M^{*}: A,
\end{gathered}
$$

in which $M^{*}=L^{*-1}$ is the inverse of $2 \mathrm{D}$ Hill's tensor under plane strain condition.

For the transition from a Cartesian coordinate to a cylindrical coordinate, we know

$$
\left[\begin{array}{cc}
\sigma_{1} & 0 \\
0 & \sigma_{2}
\end{array}\right] \longleftrightarrow\left[\begin{array}{ll}
\sigma_{1} \cos ^{2} \theta+\sigma_{2} \sin ^{2} \theta & \left(\sigma_{2}-\sigma_{1}\right) \sin \theta \cos \theta \\
\left(\sigma_{2}-\sigma_{1}\right) \sin \theta \cos \theta & \sigma_{1} \sin ^{2} \theta+\sigma_{2} \cos ^{2} \theta
\end{array}\right]
$$

Therefore, in the cylindrical coordinate, the matrix $J$ that represents the antisymmetric component of the strain can be writes as

$$
J=\left[\begin{array}{cc}
1 & 0 \\
0 & -1
\end{array}\right] \longrightarrow J=\left[\begin{array}{cc}
\cos 2 \theta & -\sin 2 \theta \\
-\sin 2 \theta & -\cos 2 \theta
\end{array}\right] .
$$

We note the principal directions of $A$ and choose them as the $(x, y)$ axes:

$$
A=\left[\begin{array}{cc}
\sigma_{1} & 0 \\
0 & \sigma_{2}
\end{array}\right]=\frac{\sigma_{1}+\sigma_{2}}{2} \delta+\frac{\sigma_{1}-\sigma_{2}}{2} J
$$


in which $\delta$ represents the symmetric part and $J$ represents the antisymmetric part.

On the cavity wall, we have

$$
\begin{gathered}
\sigma_{r r}=e_{r} \cdot \sigma \cdot e_{r}=\frac{\sigma_{1}+\sigma_{2}}{2}+\frac{\sigma_{1}-\sigma_{2}}{2} \cos 2 \theta, \\
\sigma_{r \theta}=e_{\theta} \cdot \sigma \cdot e_{r}=-\frac{\sigma_{1}-\sigma_{2}}{2} \sin 2 \theta .
\end{gathered}
$$

in which $e_{r}$ and $e_{\theta}$ are the unit vectors along the radial and tangential directions, respectively.

The combination of the 3 fields $\sigma^{1}, \sigma^{2}, \sigma^{3}$ yields,

$$
\begin{gathered}
\sigma_{r r}=-\frac{E}{1+\nu}\left[a_{1}+\left(3 a_{2}+2 a_{3}\right) \cos 2 \theta\right], \\
\sigma_{r \theta}=-\frac{E}{1+\nu}\left(3 a_{2}+a_{3}\right) \sin 2 \theta .
\end{gathered}
$$

By comparing two sets of equations and ensuring that they satisfy all possible $\theta$, we have

$$
\left\{\begin{array}{l}
a_{1}=-\frac{1+\nu}{E} \frac{\sigma_{1}+\sigma_{2}}{2} \\
a_{2}=\frac{1+\nu}{E} \frac{\sigma_{1}-\sigma_{2}}{2} \\
a_{3}=-\frac{1+\nu}{E}\left(\sigma_{1}-\sigma_{2}\right) .
\end{array}\right.
$$

Similarly, the displacement field is $u=u^{1}+u^{2}+u^{3}$ on $r=R$ :

$$
\begin{gathered}
u_{r}=a_{1} R+a_{2} R \cos 2 \theta+2 a_{3}(1-\nu) R \cos 2 \theta, \\
u_{\theta}=a_{2} R \sin 2 \theta-a_{3}(1-2 \nu) R \sin 2 \theta .
\end{gathered}
$$

If we write, on $r=R, x=R e_{r}$, then we have

$$
\begin{gathered}
B=b^{0} \delta+b^{1} J=b^{0}\left[\begin{array}{cc}
1 & 0 \\
0 & -1
\end{array}\right]+b^{1}\left[\begin{array}{cc}
\cos 2 \theta & -\sin 2 \theta \\
-\sin 2 \theta & -\cos 2 \theta
\end{array}\right] \\
u_{r}=\left(R B \cdot e_{r}\right)_{r}=R b^{0}+R b^{1} \cos 2 \theta \\
u_{\theta}=\left(R B \cdot e_{r}\right)_{\theta}=-R b^{1} \sin 2 \theta
\end{gathered}
$$

Comparing the previous two sets of equations, we have

$$
\left\{\begin{array}{l}
b_{0}=a_{1} \\
b^{1}=a_{2}+2 a_{3}(1-\nu) \\
b^{1}=-a_{2}+a_{3}(1-2 \nu) .
\end{array}\right.
$$


So we can obtain

$$
\left\{\begin{array}{l}
b_{0}=a_{1}=-\frac{1+\nu}{E} \frac{\sigma_{1}+\sigma_{2}}{2}=-\frac{1+\nu}{2 E} \delta: A \\
b^{1}=a_{2}+2 a_{3}(1-\nu)=\frac{1+\nu}{E}(4 \nu-3) \frac{\sigma_{1}-\sigma_{2}}{2} .
\end{array}\right.
$$

By substituting Eq. A.24 into the expression for $B$ (Eq. A.9), we can deduce that,

$$
M^{*}=L^{*-1}=\frac{1+\nu}{E}[(3-4 \nu) I-(1-2 \nu) \delta \otimes \delta],
$$

in which $I_{i j k l}=\frac{1}{2}\left(\delta_{i k} \delta_{j l}+\delta_{i l} \delta_{j k}\right)$.

\section{References}

[1] A. Pouya, C. Zhu, C. Arson, Micro-macro approach of salt viscous fatigue under cyclic loading, Mechanics of Materials 93 (2016) 13-31.

[2] D. Munson, P. Dawson, Constitutive model for the low temperature creep of salt (with application to wipp), Tech. rep., Sandia Labs., Albuquerque, NM (USA) (1979).

[3] Y. Lu, D. Elsworth, L. Wang, A dual-scale approach to model timedependent deformation, creep and fracturing of brittle rocks, Computers and Geotechnics 60 (2014) 61-76.

[4] C. Zhu, C. Arson, A model of damage and healing coupling halite thermo-mechanical behavior to microstructure evolution, Geotechnical and Geological Engineering 33 (2) (2014) 389-410.

[5] P. Bérest, J. Bergues, B. Brouard, J. Durup, B. Guerber, A salt cavern abandonment test, International Journal of Rock Mechanics and Mining Sciences 38 (3) (2001) 357-368.

[6] C. Zhu, A. Pouya, C. Arson, Micro-macro analysis and phenomenological modelling of salt viscous damage and application to salt caverns, Rock Mechanics and Rock Engineering (2015) 1-14doi:10.1007/s00603015-0832-9.

[7] J. Deng, Q. Yang, Y. Liu, Y. Pan, Stability evaluation and failure analysis of rock salt gas storage caverns based on deformation reinforcement theory, Computers and Geotechnics 68 (2015) 147-160. 
[8] L. B. Martin, R. Wolter, J. Rutqvist, K.-H. Lux, J. T. Birkholzer, Comparison of two simulators to investigate thermal-hydraulic-mechanical processes related to nuclear waste isolation in saliferous formations, Computers and Geotechnics 66 (2015) 219-229.

[9] N. L. Carter, F. D. Hansen, Creep of rock salt, Tectonophysics 92 (4) (1983) 275-333.

[10] F. Barbe, L. Decker, D. Jeulin, G. Cailletaud, Intergranular and intragranular behavior of polycrystalline aggregates. part 1: F.e. model, International Journal of Plasticity 17 (2001) 513-536.

[11] F. Havlicek, J. Kratochvil, M. Tokuda, V. Lev, Finite element model of plastically deformed multicrystal, International Journal of Plasticity 6 (3) (1990) 281-291.

[12] A. Beaudoin, P. Dawson, K. Mathur, U. Kocks, A hybrid finite element formulation for polycrystal plasticity with consideration of macrostructural and microstructural linking, International Journal of Plasticity 11 (5) (1995) 501-521.

[13] H. Takahashi, K. Fujiwara, T. Nakagawa, Multiple-slip work-hardening model in crystals with application to torsion-tension behaviors of aluminium tubes, International Journal of Plasticity 14 (6) (1998) 489-509.

[14] A. Musienko, A. Tatschl, K. Schmidegg, O. Kolednik, R. Pippan, G. Cailletaud, Three-dimensional finite element simulation of a polycrystalline copper specimen, Acta Materialia 55 (2007) 4121-4136.

[15] A. Needleman, A continuum model for void nucleation by inclusion debonding, Journal of Applied Mechanics 54 (3) (1987) 525-531.

[16] H. D. Espinosa, P. D. Zavattieri, A grain level model for the study of failure initiation and evolution in polycrystalline brittle materials. part i: Theory and numerical implementation, Mechanics of Materials 35 (2003) 333-364.

[17] J. Clayton, D. McDowell, Homogenized finite elastoplasticity and damage: theory and computations, Mechanics of Materials 36 (2004) 799824 . 
[18] Y. Gui, H. H. Bui, J. Kodikara, An application of a cohesive fracture model combining compression, tension and shear in soft rocks, Computers and Geotechnics 66 (2015) 142-157.

[19] N. Sukumar, D. J. Srolovitz, T. J. Baker, J.-H. Prevost, Brittle fracture in polycrystalline microstructures with the extended finite element method, International Journal for Numerical Methods in Engineering 56 (2003) 2015-2037.

[20] R. Becker, S. Panchanadeeswaran, Effects of grain interactions on deformation and local texture in polycrystals, Acta Metallurgica et Materialia 43 (7) (1995) 2701-2719.

[21] F. Delaire, J. Raphanel, C. Rey, Plastic heterogeneities of a copper multicrystal deformed in uniaxial tension: experimental study and finite element simulations, Acta Materialia 48 (5) (2000) 1075-1087.

[22] P. Erieau, C. Rey, Modeling of deformation and rotation bands and of deformation induced grain boundaries in if steel aggregate during large plane strain compression, International Journal of Plasticity 20 (2004) 1763-1788.

[23] F. Eberl, S. Forest, G. C. Cailletaud, T. Wroblewski, J. L. Lebrun, Finite-element calculations of the lattice rotation field of a tensile-loaded nickel-based alloy multicrystal and comparison with topographical x-ray diffraction measurements, Metallurgical and Materials Transactions A 33 (9) (2002) 2825-2833.

[24] A. Musienko, G. Cailletaud, Simulation of inter- and transgranular crack propagation in polycrystalline aggregates due to stress corrosion cracking, Acta Materialia 57 (2009) 3840-3855.

[25] A. Pouya, A finite element method for modeling coupled flow and deformation in porous fractured media, International Journal for Numerical and Analytical Methods in Geomechanics 39 (16) (2015) 1836-1852.

[26] G. Groves, A. Kelly, Independent slip systems in crystals, Philosophical Magazine 8 (89) (1963) 877-887.

[27] R. Stokes, Mechanical properties of polycrystalline sodium chloride, Brit. Ceram. Soc. Proc. 6 (1966) 187-207. 
[28] P. Senseny, F. Hansen, J. Russell, N. Carter, J. Handin, Mechanical behaviour of rock salt: phenomenology and micromechanisms, International journal of rock mechanics and mining sciences \& geomechanics abstracts 29 (4) (1992) 363-378.

[29] M. Sangid, The physics of fatigue crack initiation, International Journal of Fatigue 57 (2013) 58-72.

[30] A. Pouya, Correlation between mechanical behaviour and petrological properties of rock salt, in: The 32nd US Symposium on Rock Mechanics (USRMS), 1991, pp. 385-392.

[31] S. Kalidindi, C. Bronkhorst, L. Anand, Crystallographic texture evolution in bulk deformation processing of fcc metals, Journal of the Mechanics and Physics of Solids 40 (3) (1992) 537-569.

[32] A. Pouya, P. B. Yazdi, A damage-plasticity model for cohesive fractures, International Journal of Rock Mechanics and Mining Sciences 73 (2015) $194-202$.

[33] A. Jefferson, Plastic-damage model for interfaces in cementitious materials, Journal of Engineering Mechanics 124 (7) (1998) 775-782.

[34] C. Zhu, C. Arson, A. Pouya, Theoretical and numerical matrix-inclusion models of damage accommodation in salt subject to viscous fatigue, in: Mechanical Behavior of Salt VIII, Rapid City, South Dakota, 26-28 May $2015,2015$.

[35] A. Lewis, A. Geltmacher, Image-based modeling of the response of experimental 3d microstructures to mechanical loading, Scripta Materialia 55 (1) (2006) 81-85.

[36] Z. Schleder, J. L. Urai, Journal of Structural Geology 29 (2) (2007) 241 $-255$.

[37] GiD: The personal pre and postprocessor, International Center for $\mathrm{Nu}-$ merical Methods in Engineering (CIMNE).

[38] G. Simmons, H. Wang, Single crystal elastic constants and calculated aggregate properties: a handbook, M.I.T. Press, 1971. 
[39] K. Fuenkajorn, D. Phueakphum, Effects of cyclic loading on mechanical properties of maha sarakham salt, Engineering Geology 112 (1) (2010) $43-52$.

[40] A. K. Dutta, D. Penumadu, Hardness and modulus of individual sand particles using nanoindentation, in: Advances in Measurement and Modeling of Soil Behavior, 2007, pp. 1-10. doi:10.1061/40917(236)34.

[41] M. Dittes, J. F. Labuz, Field and laboratory testing of st. peter sandstone, Journal of Geotechnical and Geoenvironmental Engineering 128 (5) (2002) 372-380.

[42] C. Bullough, C. Gatzen, C. Jakiel, M. Koller, A. Nowi, S. Zunft, Advanced adiabatic compressed air energy storage for the integration of wind energy, in: Proceedings of the European Wind Energy Conference, EWEC 2004, London UK, 22-25 November, 2004.

[43] P. Bérest, Thermomechanical aspects of high frequency cycling in salt storage caverns, in: International Gas Union Research Conference 2011, Seoul, South Korea, Oct 2011, 2011.

[44] M. Raju, S. K. Khaitan, Modeling and simulation of compressed air energy storage in caverns: A case study of the huntorf plant, Applied Energy 89 (2012) 474-481.

[45] L. Ma, X. Liu, M. Wang, H. Xu, R. Hua, P. Fan, S. Jiang, G. Wang, Q. Yi, Experimental investigation of the mechanical properties of rock salt under triaxial cyclic loading, International Journal of Rock Mechanics and Mining Sciences 62 (2013) 34-41.

[46] M. Heap, S. Vinciguerra, P. Meredith, The evolution of elastic moduli with increasing crack damage during cyclic stressing of a basalt from mt. etna volcano, Tectonophysics 471 (2009) 153-160.

[47] R. Hill, Continuum micro-mechanics of elastoplastic polycrystals, Journal of the Mechanics and Physics of Solids 13 (2) (1965) 89-101.

[48] G. J. Dvorak, Y. A. Behei-El-Din, A. M. Wafa, The modeling of inelastic composite materials with the transformation field analysis, Modelling and Simulation in Materials Science and Engineering 2 (3A) (1994) 571586. 
[49] A. Fritsch, C. Hellmich, L. Dormieux, Ductile ductile sliding between mineral crystals followed by rupture of collagen crosslinks: Experimentally supported micromechanical explanation of bone strength, Journal of Theoretical Biology 260 (2) (2009) 230-252.

[50] P. Vena, D. Gastaldi, R. Contro, Determination of the effective elasticplastic response of metal-ceramic composites, International Journal of Plasticity 24 (3) (2008) 483-508.

[51] B. Pichler, C. Hellmich, Upscaling quasi-brittle strength of cement paste and mortar: A multi-scale engineering mechanics model, Cement and Concrete Research 41 (2011) 467-476.

[52] A. Fritsch, C. Hellmich, P. Young, Micromechanics-derived scaling relations for poroelasticity and strength of brittle porous polycrystals, Journal of Applied Mechanics 80 (2) (2013) 1-12.

[53] Y. Liu, P. P. Castaneda, Homogenization estimates for the average behavior and eld uctuations in cubic and hexagonal viscoplastic polycrystals, Journal of the Mechanics and Physics of Solids 52 (2004) 1175-1211.

[54] Y. Liu, P. Gilormini, P. P. Castaneda, Homogenization estimates for texture evolution in halite, Tectonophysics 406 (2005) 179-195.

[55] R. A. Lebensohn, J. P. Escobedo, E. K. Cerreta, D. Dennis-Koller, C. A. Bronkhorst, J. F. Bingert, Modeling void growth in polycrystalline materials, Acta Meterialia 61 (18) (2013) 6918-6932.

[56] B. Pichler, C. Hellmich, H. A. Mang, A combined fracturemicromechanics model for tensile strain-softening in brittle materials, based on propagation of interacting microcracks, International Journal for Numerical and Analytical Methods in Geomechanics 31 (2007) 111132 .

[57] T. Zeng, J. F. Shao, W. Y. Xu, Computers and Geotechnics 70 (2015) 130-137.

[58] P. Bérest, B. Brouard, Safety of salt caverns used for underground storage, Oil and Gas Science and Technology 58 (3) (2003) 361-384. 